\title{
SIGNAL PROPAGATIONS ALONG EXCITABLE CHAINS
}

\author{
ARNAUD TONNELIER*
}

\begin{abstract}
A simplified continuous-time threshold model for wave propagation in excitable media is proposed. The ability of the resulting transmission line to convey simple signals is investigated. Existence and multistability of traveling waves where two successive units share the same waveform is established. We show that, depending on the connectivity of the transmission line, an arbitrary number of distinct signals can be transmitted. The influence of model parameters (time constants, coupling strength and connectivity) on the traveling signal properties is analyzed.
\end{abstract}

Key words. Lattice dynamical system, Traveling wave, Excitable chain, Serial communication.

AMS subject classifications. 34K31, 37L60

1. Introduction. Communication relies on the ability of a medium to transmit information from one location to another. Most often, point-to-point interactions occur only locally and information transfer between distant nodes is carried out by propagating signals. The beacon towers of the Great Wall of China are probably the first effective human construction allowing the fast transmission of a message over long distances. This optical telegraphy has been revisited in the fictional world of Tolkien [38] where a signaling through the burning of pyres is used as a means of communication between two allied realms. The transmission of a warning signal (one bit) is carried out efficiently between distant nodes and constitutes the basic building block of modern digital communication. This principle applies in many natural systems where the all-or-none signal is generated by excitable units and traveling waves are the dynamical substrate of data transmission.

Nonlinear self-sustained waves, or autowaves [40], have been identified in a large class of discrete excitable media. They have been extensively studied in chemical and biological systems [43] but have also been observed in earthquake fault motion, electronic circuits, sandpile avalanche dynamics, and, more unexpectedly, in star formation $[6,31,4,15,29,36]$. Active wave propagations have a physiological role such as the control of cellular processes or the command of heart contraction [42, 27]. They also shape nervous system development [2]. Autowaves have been used in mechanical systems to realize sophisticated functions [34], or can be associated with a social behavior such as Mexican wave or swarming behavior [13, 10]. Propagation may also produce undesirable effects like arrhythmias, Leao's spreading depressions, epidemics, species invasions or wildfires [30].

Serial communication of complex messages requires the transmission of a sequence of bits in a time window, i.e., the successive and sequential activation of nodes in a limited time. However, refractoriness may freeze further excitation and the first activation may have irreversible effects as the excitability breaks down after passage of the wave. Similarly to waves of falling dominoes [11], the initial state may not be restored and no further wave can travel immediately after the first passage. Typical examples range from propagation of discharges in neuronal networks with strong synaptic depression [18], to autowaves for image processing [28, 32]. Such media allow ultrarapid one-bit processing [37] and are well suited for go/no-go decision transmission. However, they appear to be very limited in terms of information transfer. One way to overcome this issue is to endow the all-or-none process with multiple discrete

*Univ. Grenoble Alpes, Inria, CNRS, Grenoble INP, LJK, 38000 Grenoble, France. 

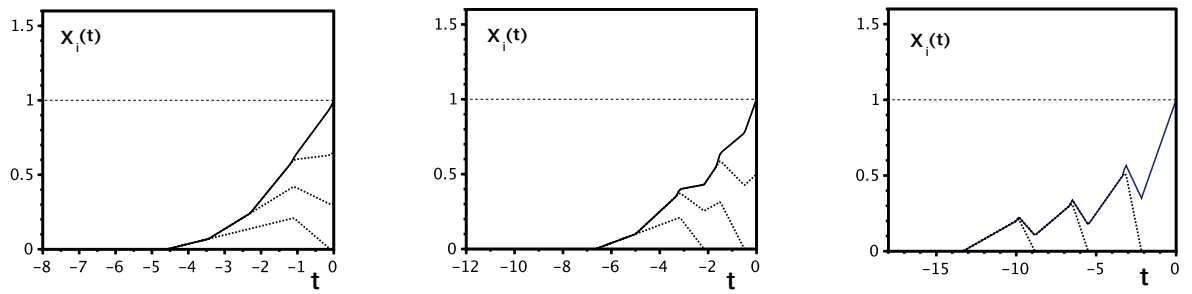

FIG. 1.1. Activity $x_{i}(t)$ of the unit $i$ at time $t$ that shows the continuous integration of incoming inputs in a transmission line with fourth-neighbor interactions. The transmission line allows the stable propagation of three distinct signals depicted in the different panels. The superposition of the weighted coupling function is indicated in dashed lines. The excitability threshold is set to 1 (horizontal straight line) and the time is shifted so that the emitting time (threshold crossing) of the unit occurs at $t=0$.

states: a set of symbols that represent or convey more than one binary digit. This strategy has been used in the semaphore telegraph developed by the French inventor Claude Chappe where each relay station has a finite set of states encoded by the shape of a mechanical system made of pivoting shutters. However, the signaling medium may not support symbolic transmission, and numerous natural systems convey only one-digit signals. Biological neural networks where neurons communicate with all-ornone action potentials or spikes are prototypical examples.

To improve the information capacity of one-bit channels, we study here an alternative strategy based on the connectivity of the transmission line. In semaphore telegraphs, the signaling stations were built in order to maximize the viewable distance, placing a tower in the line-of-sight of its two immediate neighbors. The possible benefit of an extended sight has not been explored. Rather than increasing the number of states supported by a relay station, we extend the connectivity of the transmission line so that a station can send a message beyond its closest neighbor. We think that this process may be used in natural systems for the rapid processing of simple tasks. The question is whether it would be possible to design a network that goes beyond go/no-go tasks (two-alternative choice) in a one-bit propagation framework. Mathematically, the answer relies on the multistability of signal propagations; a given transmission line supports propagating signals with different wave speeds or different waveforms. This issue is illustrated in Fig. 1.1 where an excitable transmission line with fourth-neighbor interactions shows the propagation of three distinct signals (details given in the next section). Each message is represented by a signal and the selection is made by initial conditions. In the next section, section 2, we propose a simplified excitable model for signal propagation in one-dimentional lattices. In section 3, we study the existence and stability of propagating signals where the time course of two consecutive units is shifted in time. Conditions for the coexistence of several waves are investigated. A complete analysis of the second-neighbor coupling model is conducted in section 4 and third-neighbor interactions are briefly discussed. In section 5, we conclude with a discussion.

2. An excitable lattice model. This paper deals with propagation in onedimensional excitable lattices where each site is described by a time-dependent activity, $x_{i}(t)$, where $i \in \mathcal{Z}$ is the index of the unit in the chain and $t \geq 0$ is the time. 
A unit is said to be activated when it reaches a sufficient level of activity, i.e., when the excitability threshold is crossed that reads as $x_{i}(t) \geq 1$ (we set the threshold to unity). When the $i$ th site is activated, a signal is emitted at time $t_{i}$ (where $t_{i}$ is the smallest time such that $x_{i}(t) \geq 1$ ) and is sent to the neighboring nodes. After its activation, the unit remains quiescent. The nodes are assumed to interact through a fixed coupling function $u(t)$ weighted by a scalar so that

$$
x_{i}(t)=\alpha \sum_{j} w_{i j} u\left(t-t_{j}\right),
$$

where $w_{i j}$ is the strength of the connection from $j$ to $i$ and $\alpha>0$ is the global coupling strength. The function $u$ is a fixed connectivity-driven activity that describes the trajectory initiated by the arrival of a signal.

Equation (2.1) models the two-fold dynamic process of accumulation (subthreshold dynamics) and redistribution (signal emitting) that has been identified in excitable systems where each unit integrates incoming signals and then dissipates energy back to the medium. Simplified versions of (2.1) have been used to probe self-organized criticality [4] and to explore the dynamics of earthquake faults [22], sandpile models or Abelian avalanches $[9,15]$. System $(2.1)$ describes the dynamics of spiking neurons [17], or may correspond to an electronic transmission line of switching devices [1]. It can also be obtained as an approximation of wave initiation in diffusively coupled excitable systems [21]. In (2.1), we neglect transmission delays and implicitly assume that they occur at a time scale significantly shorter than the signal transmission itself. The spatial interactions are assumed to be homogeneous and we consider a regular and uniform lattice such that

$$
w_{i j}=w(|i-j|) .
$$

We denote $w_{j}=w_{i, i-j}$ and $n$ the connection length, i.e., $w_{j}=0$, for $j>n$. The weights are normalized so that

$$
\sum_{j}\left|w_{j}\right|=1
$$

The case $n=1$ describes nearest-neighbor interactions, $n=2$ represents secondneighbor coupling and greater values of $n$ are used for higher lattices, i.e., far-neighbor interactions. Observe that pure second-neighbor interactions $\left(n=2\right.$ and $\left.w_{1}=0\right)$ result in a set of two nonconnected chains with nearest-neighbor interactions.

Excitable lattice models frequently use jump-like interactions where the coupling function is modeled by the Heaviside step function (that gives the Dirac delta function in the associated differential system). In contrast, we consider a coupling function $u$ having a temporal structure with an increasing phase followed by a decreasing one. We use the triangular function

$$
u(t)=\left\{\begin{array}{cl}
t / \tau_{1} & , 0 \leq t \leq \tau_{1} \\
1+\left(\tau_{1}-t\right) / \tau_{2} & , \tau_{1} \leq t \leq \tau_{1}+\tau_{2} \\
0 & , \text { otherwise }
\end{array}\right.
$$

where $\tau_{1}$ and $\tau_{2}$ are the rise time and the decay time, respectively, and $u$ is normalized so that $\max _{t} u(t)=1$. Qualitative results obtained in this paper do not depend on the precise expression of $u$ but rather on its global shape: a rising period of duration $\tau_{1}$ followed by a decreasing one of duration $\tau_{2}$. A time rescaling allows us to consider 
the ratio $\gamma=\tau_{1} / \tau_{2}$ as the free parameter but in order to have a transparent discussion on the role of the two characteristic times we will use both notations.

In a previous work [39], we studied an excitable lattice coming from neural modeling where the integral formulation of the model leads to a coupling function $u$ described by piecewise exponential functions. The model captures interesting properties of spiking neural networks but does not allow the in-depth analysis of the dynamics. Conditions of existence and multistability of traveling waves have not been investigated analytically and many results are based on numerical simulations.

Propagation of binary signals along the chain emerges as one unit exceeds threshold and causes neighboring units to do so in turn, thereby initiating a cascade of events that propagates through the transmission line. The successive activation of nodes relies on the existence of an increasing sequence $\left(t_{i}\right)$ where, in the simplest case, the time delay between the emitting times of two successive sites is constant: $t_{i+1}-t_{i}=1 / c$, where $c$ is the transmission speed. Such solutions will be referred to as simple, or regular, signal transmissions. More complex propagating patterns may exist with periodic, quasi-periodic or chaotic sequences $\left(t_{i}\right)$ but their analysis is left for future work. We ask the following questions: How is the signaling process related to the connectivity of the chain, $\left(w_{j}\right)$, to the global coupling strength $\alpha$ and to the time constants, $\tau_{1}, \tau_{2}$ ? What is the robustness of propagation? Is there coexistence between multiple signals?

3. Simple signals. A simple signal is defined by the sequence of emitting times $t_{i}=i / c+t_{0}$ where the translational invariance of the solution allows us to fix $t_{0}=0$. The transmitted signal is of the form $x_{i}(t)=X(t-i / c)$ where $X$ is the waveform. Let $\xi=t-i / c$ be the traveling wave coordinate. The waveform may be expressed as

$$
X(\xi)=\alpha \sum_{j=1}^{n} w_{j} u(\xi+j / c)
$$

for $\xi \leq 0$. Any solution of (3.1) will also solve (2.1) if the threshold is reached at $\xi=0$ and not before, i.e., not for $\xi<0$.

The threshold condition reads

$$
\alpha \sum_{j=1}^{n} w_{j} u(j / c)=1 .
$$

Nonlinear equation (3.2) only provides candidate solutions. If there exists a solution $c$ of (3.2) satisfying the inequality constraint (solvability or admissibility criterion)

$$
X(\xi)<1 \text {, for } \xi<0,
$$

then there exists a traveling wave solution of (2.1). The admissibility criterion (3.3) is fulfilled only if $X^{\prime}(0)>0$ which gives the necessary condition

$$
\sum_{j=1}^{n} w_{j} u^{\prime}(j / c)>0
$$

where the strict inequality holds from the piecewise constant nature of $X^{\prime}$ (otherwise the threshold is reached before $\xi=0)$. A wave speed, solution of (3.2), defines a unique waveform given by (3.1). The issue of coexistence of traveling signals relies on the existence of multiple solutions of (3.2) with respect to the wave speed, $c$, for fixed network parameters, $\alpha,\left(w_{j}\right)$ and $\gamma$. 
3.1. Stability of signals. We consider small perturbations of the emitting times $t_{j}=j / c+\epsilon_{j}$, where $\left|\epsilon_{j}\right| \ll 1$. The asymptotic stability of regular signals reads as $\epsilon_{j} \rightarrow 0$ as $j \rightarrow \infty$. The perturbed propagating activity, $y_{i}(t)$, satisfies

$$
y_{i}(t)=\alpha \sum_{j=1}^{n} w_{j} u\left(t-(i-j) / c-\epsilon_{i-j}\right)
$$

Expanding to the first order in $\epsilon_{i}$ and writing the threshold condition for $y_{i}$ leads to the linear equation

$$
\sum_{j=1}^{n} w_{j}\left(\epsilon_{i}-\epsilon_{i-j}\right) u^{\prime}(j / c)=0 .
$$

Substitution of a solution of the form $\epsilon_{i}=\lambda^{i}$ gives the characteristic equation

$$
\sum_{j=1}^{n} w_{j}\left(1-\lambda^{-j}\right) u^{\prime}(j / c)=0
$$

Multiplying by $\lambda^{n}$, we obtain the polynomial equation

$$
P(\lambda)=\sum_{j=0}^{n} a_{j} \lambda^{j}=0
$$

where the coefficients $\left(a_{j}\right)$ are given by

$$
\begin{aligned}
a_{j} & =-w_{n-j} u^{\prime}((n-j) / c), \quad j=0, \ldots, n-1, \\
a_{n} & =\sum_{j=1}^{n} w_{j} u^{\prime}(j / c) .
\end{aligned}
$$

Due to the translational invariance of traveling wave solutions, $\lambda=1$ is always a root of the characteristic equation. Let $Q$ be the polynomial

$$
P(\lambda)=(\lambda-1) Q(\lambda) .
$$

We have

$$
Q(\lambda)=\sum_{j=0}^{n-1} b_{j} \lambda^{j}
$$

where

$$
b_{j}=-\sum_{k=0}^{j} a_{k}=\sum_{k=n-j}^{n} w_{k} u^{\prime}(k / c) .
$$

The traveling wave is asymptotically stable if and only if $Q(\lambda)$ is a Schur polynomial: all roots of $Q$ lie in the interior of the unit circle. From the classical result of Eneström and Kakeya (if $\mu_{n}>\mu_{n-1}>\mu_{n-2}>\ldots>\mu_{0}>0$ then, for $|z| \geq 1, \sum_{k=0}^{n} \mu_{k} z^{k} \neq 0$ ) a sufficient condition for Schur stability is $b_{n-1}>b_{n-2}>\ldots>b_{0}>0$ that reads as

$$
w_{j} u^{\prime}(j / c)>0, \quad j \in\{1, \ldots, n\} .
$$


For purely excitatory chains $\left(w_{j}>0\right)$, condition (3.7) is fulfilled when all the emitting times belong to the rising phase of the coupling function. This can be simply rewritten as

$$
c \tau_{1}>n
$$

Notice that, from (3.2), we have

$$
\frac{d \alpha}{d c}=\frac{\alpha^{2}}{c^{2}} \sum_{j=1}^{n} j w_{j} u^{\prime}(j / c) .
$$

Thus, solutions satisfying (3.7) are related to signals with a velocity that increases with the global coupling strength, $\alpha$.

3.2. Fast signal transmission. Let us assume that the transmission speed is sufficiently large so that (3.8) is satisfied. This case will be referred to as fast signal transmission. We have $u(j / c)=j /\left(c \tau_{1}\right), j=1, \ldots, n$, and, from (3.2), the transmission speed is given by:

$$
c=\frac{\alpha}{\tau_{1}} \sum_{j=1}^{n} j w_{j}
$$

which shows the bilinearity of the propagation velocity with respect to the first moment of the weight distribution and the coupling strength $\alpha$. This scaling property is reminiscent of the speed law found for neural waves [26] and is related to the linear behavior of the coupling function $u(t)$ for $t<\tau_{1}$.

In the fast transmission limit, $c \gg 1$, the wave speed can be derived analytically for a wider class of coupling functions, not limited to piecewise linear functions. Let us consider, temporarily, an arbitrary coupling function $u$ and let $p$ be the first nonnull derivative of $u$ at 0 . From (3.2) and using

$$
u(j / c)=j^{p} /\left(p ! c^{p}\right) u^{(p)}(0)+O\left(1 / c^{p+1}\right),
$$

we get the asymptotic approximation

$$
c=\left(\alpha u^{(p)}(0) \sum_{j=1}^{n} \frac{j^{p}}{p !} w_{j}\right)^{\frac{1}{p}}+O(1 / c)
$$

that shows that the scaling law of the fast wave speed is determined, in the limit $c \gg 1$, by the smoothness of the coupling function at the onset.

In the present study, we have $p=1$ and a fast transmission is possible if the coupling strength is sufficiently large, greater than the critical value $\alpha^{*}$ given by

$$
\alpha^{*}=\frac{n}{\sum_{j=1}^{n} j w_{j}} .
$$

Calculations of the fast wave speed and of the critical coupling value $\alpha^{*}$ are presented for different weight distributions in Table 3.1. Asymptotic approximations for longrange interactions $(n \gg 1)$ are also provided. 
TABLE 3.1

Fast-signal speed for different connectivities of the transmission line. Nonnormalized weights $\left(w_{j}\right)$ are presented in the first row. The normalization coefficient, denoted $|w|$, is given in the second row. The analytical expression of the wave speed is provided in the third row. The asymptotic behaviors, as $n \rightarrow \infty$, of the wave speed and of the critical coupling coefficient, $\alpha^{*}$ (above which fast signals exist), are given in the fourth and fifth rows, respectively. The term $H_{n}$ denotes the nth harmonic number and $H_{n, 2}$ is the generalized harmonic number in power 2.

\begin{tabular}{|c|c|c|c|c|}
\hline$\left(w_{j}\right)_{j=1, \ldots, n}$ & $(1)_{j}$ & $(1 / j)_{j}$ & $\left(1 / j^{2}\right)_{j}$ & $\left(a^{j}\right)_{j}$ \\
\hline$|w|$ & $n$ & $H_{n}$ & $H_{n, 2}$ & $\left(a-a^{n+1}\right) /(1-a)$ \\
\hline$c$ & $\frac{\alpha(n+1)}{2 \tau_{1}}$ & $\frac{\alpha n}{\tau_{1} H_{n}}$ & $\frac{\alpha H_{n}}{\tau_{1} H_{n, 2}}$ & $\frac{\alpha\left(n a^{n+1}-(n+1) a^{n}+1\right)}{\tau_{1}(1-a)\left(1-a^{n}\right)}$ \\
\hline$c(n \gg 1)$ & $\frac{\alpha n}{2 \tau_{1}}$ & $\frac{\alpha n}{\tau_{1} \ln n}$ & $\frac{6 \alpha \ln n}{\pi^{2} \tau_{1}}$ & $\frac{\alpha}{\tau_{1}(1-a)}$ \\
\hline$\alpha *(n \gg 1)$ & 2 & $\ln n$ & $\frac{\pi^{2} n}{6 \ln n}$ & $n(1-a)$ \\
\hline
\end{tabular}

From (3.5), the stability of fast signals is determined by the characteristic equation

$$
\sum_{j=0}^{n-1} \sum_{k=n-j}^{n} w_{k} \lambda^{j}=0
$$

for $n>1$ (the case $n=1$ will be discussed later). The stability does not depend on the value of the wave speed and, from (3.7), fast waves are always asymptotically stable in excitatory transmission lines. It should be noted that, for constant weights, the characteristic equation is given by

$$
1+2 \lambda+3 \lambda^{2}+\ldots+n \lambda^{n-1}=0
$$

where the zeros are given by the non-unitary roots of $n \lambda^{n+1}-(n+1) \lambda^{n}+1$. A straightforward study reveals that there exists a non-unitary real root if and only if $n$ is odd and, in this case, the real root is simple and lies in the interval $]-1,0[$. Other roots are located inside the unit circle.

3.3. Classification of signals. Regular traveling signals persist as parameters change, defining branches of solutions that can be classified according to the occurrence of the emitting times $t_{i}=i / c, i=1, \ldots, n$, with respect to the two time constants $\tau_{1}$ and $\tau_{2}$. More precisely, the location of $c \tau_{1}$ and $c \tau_{1}+c \tau_{2}$ with respect to the first $n$ natural numbers determines all types of signals. There are $C_{n+2}^{2}$ choices to place these two characteristic values in $n+1$ intervals and, therefore, there exist $(n+2)(n+1) / 2$ classes of propagating waves. Let $p$ be the number of emitting times less than $\tau_{1}$ and let $q$ be the number of events that fall into the interval $] \tau_{1}, \tau_{1}+\tau_{2}$ [ that define a $(p, q)$-wave type, or $(p, q)$-signal type (for $q=0$ we simply write $p$-wave, or $p$-signal, in the following). For instance, a transmission line with $n=2$ supports up to six different classes of signals: $(2,0),(1,1),(1,0),(0,2),(0,1)$, and $(0,0)$. However, some classes may not be relevant in cases where the associated signals cannot propagate along the line, such as the trivial class $(0,0)$. In our terminology, waves such that one event $t_{i}$ equals $\tau_{1}$ or $\tau_{1}+\tau_{2}$ do not belong to a particular class. They define nonrobust solutions that disappear as one parameter is perturbed and may be seen as transitory solutions between two distinct classes. However, most results can be directly used to characterize these waves (except the stability analysis due to the nondifferentiability of such signals).

We have $p=\left\lfloor c \tau_{1}\right\rfloor$ and $p+q=\left\lfloor c \tau_{1}+c \tau_{2}\right\rfloor$. There are $n-(p+q)$ events greater than $\tau_{1}+\tau_{2}$ that play no role in the propagation since their effect on the wave dynamics 


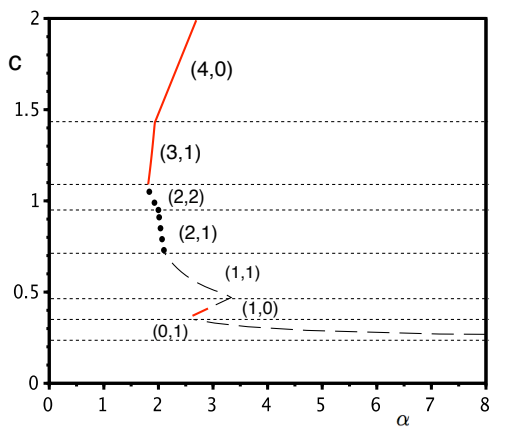

FIG. 3.1. Velocity of simple signals along an excitable chain as a function of the global coupling strength. Parameters are $\tau_{1}=2.8, \tau_{2}=1.4$ and $n=4$. Connections are given by $w_{i}=0.7^{i} /|w|$, $i=1, \ldots, 4$. The dotted horizontal straight lines separate the different wave types and are defined by $c=i / \tau_{1}$ and $c=i /\left(\tau_{1}+\tau_{2}\right), i=1, \ldots, 4$. In each zone, the class of the corresponding signals is indicated. Solid red lines stand for stable waves and dotted lines indicate unstable signals. Longdashed lines denote traveling wave candidates that do not satisfy the solvability condition.

has vanished at the threshold-crossing time (see (3.2)). For $p=n$, we obtain the fast-transmission signal previously described. The case $q=0$ defines a set of waves, parametrized by $p$, that are similar to the fast signal if we consider $p$ as the effective connection range, i.e, the weights $w_{i}, i=n-p+1, \ldots, n$, do not play a role in the propagation of such signals. In excitatory networks, the associated waveforms are strictly increasing (see (3.1)).

Within a given class, the derivatives $u^{\prime}\left(t_{i}\right)$ remain constant and the stability of waves within a class is invariant. However, the admissibility condition (3.3) may be fulfilled only by a limited number of waves within a class and some candidate waveforms can cross the excitability threshold more than once while a change in parameter values allows them to be admissible again. In the general case, the existence of waves in a given class crucially depends on the connectivity and simple conditions on parameters are difficult to obtain. In excitatory networks, a necessary condition for signal transmission is that at least one event time is lower than $\tau_{1}$ (see (3.4)). This gives $c_{\text {min }}=1 / \tau_{1}$ as a lower bound for the wave speed and reduces the maximal number of classes where admissible traveling wave solutions can be found to $n(n+1) / 2$.

Let us now assume that the time constants ratio $\gamma$ is fixed. The different waves that may propagate along the transmission line are found using the wave speed as a free parameter. For $c$ sufficiently large, all events $\left(t_{i}\right)$ fall into the increasing part of the coupling function. This case corresponds to the fast-transmission class previously introduced that is obtained when the coupling strength is sufficiently large (greater than $\alpha^{*}$ given by (3.11)). As $c$ changes, other types of waves are found. This is illustrated in Fig. 3.1, where we plot the speed curve $c$ as a function of the global strength $\alpha$ for an excitable chain with $\gamma=2\left(\tau_{1}=2.8, \tau_{2}=1.4\right)$ and $n=4$. The nonnormalized connections are given by $w_{i}=0.7^{i}$. The class-transition diagram $(4,0) \rightarrow(3,1) \rightarrow(2,2) \rightarrow(2,1) \rightarrow(1,1) \rightarrow(1,0) \rightarrow(0,1)$ is observed as the signal velocity, $c$, decreases. Note that, for the transmission line considered here, the two classes $(1,1)$ and $(0,1)$ do not contain admissible signals. 
Let us consider a $(p, q)$-signal where $p \geq 1$ and $q \neq 0$. As the velocity $c$ decreases, another wave type is reached and, depending on $\gamma=\tau_{1} / \tau_{2}$, there are three eventualities. For $\gamma<p / q$, the time event $t_{p}$ leaves the interval where $u$ increases to enter the interval where $u$ decreases. This defines a new signal type that belongs to the $(p-1, q+1)$ class. When $\gamma>p / q$, the time event $t_{p+q}$ becomes greater than $\tau_{1}+\tau_{2}$, defining a $(p, q-1)$-wave type. In the particular case where $\gamma=p / q$, we obtain a $(p-1, q)$-signal. For $q=0$, a $p$-signal becomes a $(p-1,1)$-wave as $c$ decreases. Iteration of this process leads to a maximum of $2 n$ classes. This maximum is reached when $\gamma$ is an irrational number otherwise the number of classes is reduced by one each time that there exists $(p, q)$ such that $p \tau_{2}=q \tau_{1}$. In Fig. 3.1, we find seven different types of signals. We have $\gamma=2$, so the $(3,1)$-wave becomes a $(2,2)$-signal as $c$ reaches the critical value $c=3 / \tau_{1} \approx 1.07$. The $(2,2)$-signal gives a $(2,1)$-wave type as $c=4 /\left(\tau_{1}+\tau_{2}\right) \approx 0.95$ that subsequently becomes a $(1,1)$ signal for $c=2 / \tau_{1}=3 /\left(\tau_{1}+\tau_{2}\right) \approx 0.71$. One may observe the existence of an $\alpha$-range $(\subset(2.5,3))$ where bistability occurs between fast transmission (4-signals for $c>4 / \tau_{1} \approx 1.42$ ) and 1-signal (for $\left.c \in\right] 1 / \tau_{1}, 2 /\left(\tau_{1}+\tau_{2}\right)[\approx] 0.35,0.47[$ ).

From (3.2), the speed of a $(p, q)$ traveling signal is given by

$$
c=\frac{1}{\tau_{1}} \frac{\sum_{j=1}^{p} j w_{j}-\gamma \sum_{j=p+1}^{p+q} j w_{j}}{\frac{1}{\alpha}-(1+\gamma) \sum_{j=p+1}^{p+q} w_{j}}
$$

for $q \geq 1$. For $q=0$, the velocity $c$ is given by

$$
c=\frac{\alpha}{\tau_{1}} \sum_{j=1}^{p} j w_{j} .
$$

As for fast-signal transmission, one may expect that the velocity of a $(p, q)$-signal increases with $\alpha$, the global coupling strength. However, traveling signals such that

$$
\gamma \sum_{j=p+1}^{p+q} j w_{j}>\sum_{j=1}^{p} j w_{j}
$$

have a velocity that strictly decreases with $\alpha$. Note that, from (3.12), the speed of $p$-signals increases with the global coupling $\alpha$.

3.4. Multistability of signal transmissions. The coexistence, when parameters of the network are fixed, of multiple signals determines the ability of the medium to transmit different messages. In particular, the existence of stable traveling signals having different velocities may be used for a multiple-choice decision making where one decision is represented by a signal, or, potentially, by the no-propagation case. The selection of traveling signals is monitored by the initial excitation and depends on the basin of attraction of the different waves.

Before the study of the coexistence of waves, let us investigate the stability of a $(p, q)$ signal. From (3.6) and using $u^{\prime}(k / c)=0$ for $k>p+q$, the stability is determined by the roots of the characteristic polynomial

$$
\begin{aligned}
Q(\lambda) & =\sum_{i=n-(p+q)}^{n-1} b_{i} \lambda^{i}, \\
& =\lambda^{n-(p+q)} \tilde{Q}(\lambda)
\end{aligned}
$$


where

$$
\tilde{Q}(\lambda)=\sum_{i=0}^{p+q-1} b_{n-(p+q)+{ }_{i}} \lambda^{i} .
$$

Using (3.6), the $i$ th coefficient, $b_{n-(p+q)+i}$, can be rewritten as

$$
\begin{aligned}
& -\frac{1}{\tau_{2}} \sum_{j=0}^{i} w_{p+q-j}, \text { if } i<q, \\
& \frac{1}{\tau_{1}} \sum_{j=q}^{i} w_{p+q-j}-\frac{1}{\tau_{2}} \sum_{j=0}^{q-1} w_{p+q-j} \text { if } i \geq q .
\end{aligned}
$$

Let us assume that $c(\alpha)$ is strictly decreasing. Condition (3.13) reads

$$
\tilde{Q}(1)<0
$$

The necessary condition (3.4) for the existence of a traveling signal can be rewritten for a $(p, q)$-signal as

$$
\sum_{j=1}^{p} w_{j}>\gamma \sum_{j=p+1}^{p+q} w_{j}
$$

which reads as $b_{n-1}>0$ and therefore $\lim _{\lambda \rightarrow+\infty} \tilde{Q}(\lambda)=+\infty$. Using (3.14), there exists a real root of $\tilde{Q}$ greater than one that shows that $(p, q)$-signals with decreasing velocities (with respect to $\alpha$ ) are unstable. Therefore, the possible multistability of propagating signals has to be investigated in regions where the signal speed increases with the coupling strength $\alpha$.

Conversely, one may wonder if regimes where $d c / d \alpha>0$ are associated with stable signals. Although this is true when $n=2$ (see section 4), it turns out to be false in general. A counterexample is provided by the excitable chain where $n=4, \tau_{1}=1$, $\tau_{2}=0.4$, and $\left(w_{i}\right)_{i=1, \ldots, 4}=(0.15,0.35,0.35,0.15)$. We easily show that the $(3,1)$-wave is admissible and has a velocity that increases with $\alpha$. Its stability is determined by the polynomial

$$
Q(\lambda)=0.475 \lambda^{3}+0.325 \lambda^{2}-0.0250 \lambda-0.3750,
$$

where the roots are

$$
\lambda_{ \pm}=-0.7212 \pm 0.7218 i \text { and } \lambda_{0}=0.7583
$$

Since $\left|\lambda_{ \pm}\right|>1$ the $(3,1)$-traveling signal is unstable.

As observed in Fig. 3.1, different $p$-signals may coexist ( $p=4$ and $p=1$ for parameters of Fig. 3.1). Let us now restrict our attention to the existence and stability of $p$ signals, $p \in\{1, \ldots, n\}$, in excitatory transmission lines. As already observed, the signal speed increases with $\alpha$. In addition, the classical Eneström-Kakeya theorem directly applied to the associated characteristic polynomial and a $p$-signal is always stable. A $p$-wave exists if the first $p$ events are less than $\tau_{1}$ while the remaining events are greater than $\tau_{1}+\tau_{2}$. For $p \leq n-1$, this is possible only when $(p+1) /\left(\tau_{1}+\tau_{2}\right)>$ $p / \tau_{1}$, which reads as

$$
\gamma>p
$$


This is shown in Fig.3.1 where $1<\gamma \leq 2$ so that 1-signals can propagate but not 2 -signals. For $p=n$ (fast-transmission case), no condition is required on the time constants (see the existence of 4-signals in Fig. 3.1). Notice that the existence of a $p$-signal for $2 \leq p \leq n-1$ implies the existence of a $(p-1)$-signal (provided that the admissibility conditions are satisfied).

Let us consider the two transmission speeds

$$
c_{p}^{l}=\frac{p}{\tau_{1}}, \quad \text { and } \quad c_{p}^{r}=\frac{p+1}{\tau_{1}+\tau_{2}} .
$$

Assuming (3.16), the velocity of a $p$-signal belongs to the interval $] c_{p}^{l}, c_{p}^{r}[$ and the corresponding $\alpha$-range is given by $\left.D_{p}=\right] \alpha_{2 p-1}, \alpha_{2 p}[$, where

$$
\alpha_{2 p-1}=\frac{p}{\sum_{j=1}^{p} j w_{j}}, \quad \text { and } \quad \alpha_{2 p}=\frac{p+1}{\left(1+\frac{1}{\gamma}\right) \sum_{j=1}^{p} j w_{j}},
$$

where $p \in\{1, \ldots, n\}$ and we set $\alpha_{2 n}=+\infty$. Condition (3.16) ensures that $\alpha_{2 p}>$ $\alpha_{2 p-1}$.

3.4.1. Branching points of the speed curve. The network exhibits multiple stable propagating signals when, for fixed parameters, two different $p$-signals exist; i.e., a nonempty intersection can be found between two different $\alpha$-intervals $D_{p}$. A necessary condition is that the sequence $\left(\alpha_{k}\right)$ defined by (3.18) is not increasing; i.e., there exists an index $q<\gamma$ such that $\alpha_{2 q}>\alpha_{2 q+1}$ that yields the inequality

$$
\gamma(q+1) w_{q+1}>\sum_{j=1}^{q} j w_{j}
$$

where the strict inequality ensures that the intersection is not reduced to a point. Condition (3.19) implies the existence of a turning point of the speed curve, i.e., a fold of the $c(\alpha)$ curve, as observed in Fig. 3.1 for $q=1$. Several turning points of the speed curve are obtained if inequality (3.19) is satisfied for different $q$ values.

When the speed curve folds (in the $c-\alpha$ plane), it forms an additional branch of solutions and the point $\left(\alpha_{2 q}, c_{q}^{r}\right)$ defines a branching point of (3.2) (that will be referred to as a right turning point or right folding) where one branch (for $c<c_{q}^{r}$ ) is associated with the $q$-signal. The other branch corresponds to an unstable traveling signal (since $d c / d \alpha<0)$ that subsequently bifurcates, defining the left turning point $\left(\alpha_{2 q+1}, c_{q+1}^{l}\right)$ associated with the $(q+1)$-signal for $\gamma>q+1$. Otherwise another branching point, not related to a $p$-signal type, occurs at larger $c$ values (see the turning point in Fig. 3.1 at the transition $(2,2) \rightarrow(3,1))$. The possible multistability of signals is associated with the existence of branching bifurcations as $\alpha$ changed. Other bifurcation scenarios will be discussed in section 4 .

In the context of signal transmission, one may wonder, What is the maximal number of stable waves supported by the medium? We ask the question, What are the conditions on transmission line parameters for the simultaneous existence of $p$-signals for $p=$ $1, \ldots, n$ ? Let us assume in the following that

$$
\gamma>n-1
$$

Inequality (3.20) ensures that each $p$-signal is a candidate solution. In addition, the co-existence, for $\alpha$ fixed, of all $p$-signals requires that each $\alpha_{2 q}$ corresponds to a 
turning point of the $c(\alpha)$ curve, i.e., (3.19) is satisfied for $q=1 \ldots n-1$ (and thus each $\left(\alpha_{2 q-1}, c_{q}^{l}\right)$ is a branching point). This gives the conditions for the following weight distributions:

- Constant weights: $w_{i}=1 / n$. Condition (3.19) is fulfilled for $q=1 \ldots n-1$ if and only if $\gamma>(n-1) / 2$. From (3.20), the points $\left(\alpha_{2 q}, c_{q}^{r}\right)$ are branching points of the speed equation (3.2) (and, subsequently, each point $\left(\alpha_{2 q-1}, c_{q}^{l}\right)$ also defines a bifurcation point). This is illustrated in Fig. 3.2(a) where $\gamma=5.5$ and $n=6$. We have $\gamma>n-1$ and the speed curve folds at each $\alpha_{k}$.

- Exponential weights: $w_{i}=a^{i}$ (nonnormalized). Condition (3.19) reads

$$
G_{q}(a)=\frac{q a^{q+1}-(q+1) a^{q}+1}{a^{q}(1-a)^{2}}<\gamma .
$$

We can easily show that $G_{q}$ is an increasing function of $q$ and the speed curve folds at each $\alpha_{2 q}$ value if and only if (3.21) is satisfied for $q=n-1$. A straightforward monotonicity analysis shows that $G_{n-1}(a)$ strictly decreases with $a$ and $\lim _{a \rightarrow 0} G_{n-1}(a)=+\infty, \lim _{a \rightarrow+\infty} G_{n-1}(a)=0$ so that there exists a critical value $a_{n}^{*}$ such that the folding condition $G_{n-1}(a)<\gamma$ is satisfied for $a>a_{n}^{*}$ (to avoid heavy notation we drop the $\gamma$-dependence). Since $G_{n-1}(1)=(n-1) / 2$ one has $a_{n}^{*}<1$. The critical value $a_{n}^{*}$ increases with $n$ and for $\gamma=O(n)$ and $n \gg 1$, the following asymptotic approximation holds:

$$
a_{n}^{*} \sim 1-\frac{1}{\sqrt{\gamma}}
$$

In Figure 3.2(b), (c) the speed curve of a transmission line where $n=6$ is plotted for $\gamma=5.5$ and for two different exponential weight distributions, $a=0.8$, and $a=0.6$, respectively. We calculate $a_{6}^{*} \approx 0.739$. In (b), we have $a>a_{6}^{*}$ so that each $\alpha_{2 q}$ is associated with a branching point. In (c), we have $a<a_{6}^{*}$ and the folding condition is satisfied only for $q=1,2,3$.

The previous conditions are necessary conditions ensuring that each $p$-wave is a candidate solution that can coexist for different $p$-values. As shown in Fig. 3.2, branches of the speed curve associated with $p$-signals, or part of the branches, do not fulfill the admissibility condition. Therefore, to guarantee the existence of signals and, subsequently, the possible multistability, the solvability condition (3.3) has to be checked. This is done in the next section.

3.4.2. Solvability condition. Let us consider regular signals $X(\xi)$ given by (3.1). The solvability condition (3.3) ensures that the threshold is not reached before $\xi=0$. The traveling signal is the weighted superposition of shifted functions $u(t+$ $j / c$ ) where the elementary function $u(t)$ admits a maximum at $t=\tau_{1}$. A necessary and sufficient condition to fulfill the solvability condition is that the (possible) local maxima of $X$, located at $\xi=\tau_{1}-j / c$ are strictly less than one.

For a $p$-signal, we have $\tau_{1}-j / c>0$ for $j \leq p$, and condition (3.3), for $p \neq n$, becomes

$$
X\left(\tau_{1}-k / c\right)<1, \quad k=p+1, \ldots, n
$$

For $p=n$, the traveling wave is admissible and corresponds to the fast-transmission signal. We assume in the following that $p \in\{1, \ldots, n-1\}$. We have $c<(p+1) /\left(\tau_{1}+\tau_{2}\right)$ and thus $\tau_{1}<(p+1) / c$, which gives $u(t-i / c)=0$ for $i \geq p+1$. In addition, 


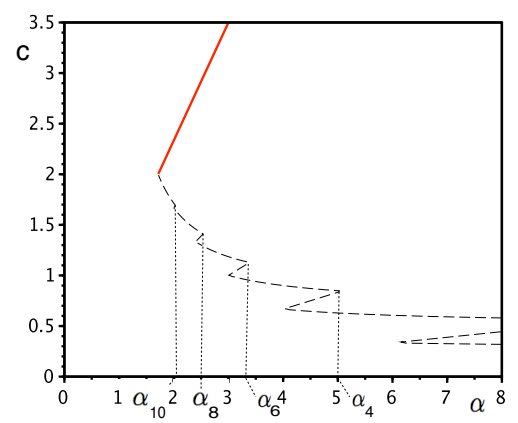

(a)

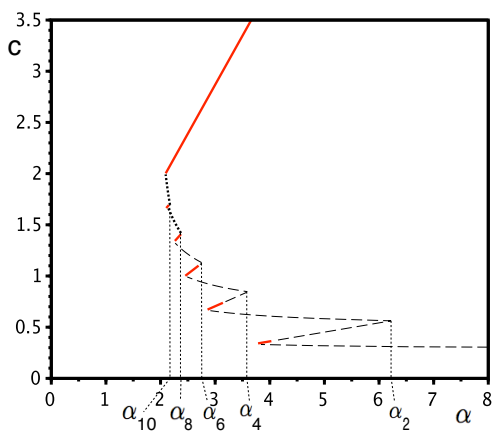

(b)

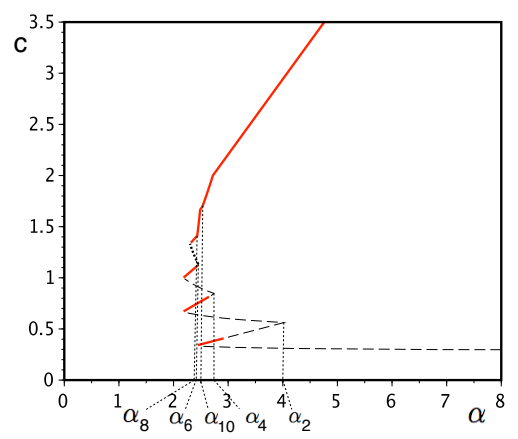

(c)

FIG. 3.2. Velocity, c, of simple signals along an excitable chain as a function of the global coupling strength, $\alpha$. Red lines denote stable propagating signals and dotted lines are for unstable waves. Long-dashed lines indicate nonadmissible solutions, i.e., solutions of (3.2) that violate (3.3). Parameters of the transmission line are $\gamma=5.5\left(\tau_{1}=3, \tau_{2} \approx 0.54\right), n=6$, and the connections are given by $w_{i}=a^{i}$ (non normalized) where (a) $a=1$, i.e., constant weights, (b) $a=0.8$, and (c) $a=0.6$. A p-signal is defined on the interval $] \alpha_{2 p-1}, \alpha_{2 p}\left[\right.$ where $p=1, \ldots, 6$. The sequence $\alpha_{2 p}$, $p=1, \ldots, 5$ is indicated. We have $a_{6}^{*} \approx 0.739$, so that, in (a) and (b), the speed curve folds at each $\alpha_{2 p}$ value whereas in (c) there is no bifurcation point at $\alpha_{2 p}$, for $p=4,5$. 
from $c>p / \tau_{1}$, it follows that $\tau_{2}<1 / c$. This implies that $\tau_{1}+1 / c>\tau_{1}+\tau_{2}$ and $u\left(\tau_{1}+i / c\right)=0$ for $i \geq 1$. Developing the left-hand side of (3.22) gives

$$
\alpha w_{k}+\alpha w_{k-1} u\left(\tau_{1}-1 / c\right)+\ldots+\alpha w_{k-p} u\left(\tau_{1}-p / c\right)<1, \quad k=p+1, \ldots, n .
$$

Using $u\left(\tau_{1}-i / c\right)=1-i /\left(c \tau_{1}\right)$ and $c \tau_{1}=\alpha \sum_{j=1}^{p} j w_{j}$, we obtain the following admissibility condition for $p$-signals:

$$
\alpha<\alpha_{p, i}^{*}, \quad i=1, \ldots, n-p,
$$

where the critical values $\alpha_{p, i}^{*}$ are given by

$$
\alpha_{p, i}^{*}=\frac{1}{w_{i}+\ldots+w_{i+p}}\left(1+\frac{p w_{i}+(p-1) w_{i+1}+\ldots+w_{i+p-1}}{w_{1}+2 w_{2}+\ldots+p w_{p}}\right) .
$$

Since a $p$-signal is defined on the interval $] \alpha_{2 p-1}, \alpha_{2 p}[$, a necessary and sufficient condition for the existence of $p$-signals is $\alpha_{p, i}^{*}>\alpha_{2 p-1}$ that gives

$$
w_{1}+\ldots+p w_{p}>w_{i+1}+\ldots+p w_{i+p}, \quad i=1, \ldots, n-p,
$$

i.e., the weighted average of the first $p$ coupling coefficients is greater than the weighted moving average.

We present the solvability condition (3.23), for the following weight distributions,

- Constant weights. Condition (3.23) cannot be fulfilled and $p$-signals do not exist $\forall p \in\{1, \ldots, n-1\}$. This is shown in Fig. 3.2(a) where branches associated with the different $p$-signals are not admissible (except for $p=n$ ).

- Exponential weights $\left(w_{i}=a^{i}\right)$. Condition (3.23) gives $a<1$. In Figure $3.2(\mathrm{~b})$, (c), we observe that each branch presents a section where the admissibility condition is satisfied.

The following remarks are directly derived from (3.23). The $(n-1)$-wave is admissible if and only if $(n-1) w_{n}<w_{1}+\ldots+w_{n-1}$. The 1-signal exists if $w_{1}>w_{i}, i=2, \ldots, n$. In a chain with increasing weights, the fast wave is the only admissible $p$-wave solution.

3.4.3. A multistability theorem. Using the solvability condition (3.23), one can find a network connectivity $\left(w_{i}\right)_{i=1, \ldots, n}$, and a time constants ratio $\gamma>n-1$ for which $n$ distinct signals propagate stably. One may wonder if there exists an $\alpha$-range for which all these waves coexist. This condition reads

$$
\bigcap_{p=1}^{n} I_{p} \neq \emptyset
$$

where $I_{p} \subset D_{p}$ is the interval where $p$-signals are admissible traveling wave solutions. We have

$$
\left.I_{p}=\right] \alpha_{2 p-1}, \min \left(\left(\alpha_{p, i}^{*}\right)_{i=1, \ldots, n-p}, \alpha_{2 p}\right)[,
$$

where, for $p=n$, we set $\alpha_{n}^{*}=\infty$, and if, for a given $p$, the right bound of the interval is lower than the left bound, the corresponding interval is assumed to be empty. A necessary condition for $(3.24)$ is $\min _{i=1, \ldots, n-p} \alpha_{p, i}^{*}>\alpha_{2 p-1}$ for $p=1, \ldots, n-1$.

A simple configuration is obtained when all $\alpha_{2 p-1}$ values, $p=1, \ldots, n$, are identical. This case corresponds to a vertical alignment of the left turning points of the speed 
curve, in the $c$ - $\alpha$ plane. Using (3.18), straightforward calculations lead to the following connectivity of the transmission line

$$
w_{i}=\frac{1}{i H_{n}}, \quad i=1, \ldots, n
$$

where the $H_{n}$ is the $n$th harmonic number and stands for the normalization coefficient. We have $\alpha_{2 p-1}=H_{n}$. We are now in position to prove the following theorem on the existence and stability of propagating signals:

TheOREM 3.1 (Multistability). Let $n-1<\gamma \leq n H_{n}-1$, and let $\left(w_{i}\right)$ be given by (3.25); then there exists a nonempty $\alpha$-interval given by

$$
\left.I_{n-1}=\right] H_{n}, \frac{n H_{n}}{(n-1)\left(1+\frac{1}{\gamma}\right)}[
$$

such that the transmission line supports the stable propagation of $n$ distinct signals with velocities given by

$$
c_{p}=\frac{\alpha p}{\tau_{1} H_{n}}, \quad p=1, \ldots, n
$$

Proof. Since we have $\gamma>n-1$, the $\alpha$-intervals where $p$-signals exist are well defined for $p=1, \ldots, n$ (see (3.18)). From (3.25) and using $k /(k+i)<1$, we find that the solvability condition (3.23) holds for each $p$-wave and the associated branch of the speed curve presents an admissible section. Note that the folding condition (3.19) becomes $\gamma>q$, which is fulfilled. From $\alpha_{2 p-1}=H_{n}$, the intersection of the $\alpha$-intervals where $p$-waves exist is given by $] H_{n}, \alpha^{*}[$ where

$$
\alpha^{*}=\min _{p=1, \ldots, n-1}\left(\alpha_{2 p}, \min _{i=1, \ldots, n-p} \alpha_{p, i}^{*}\right) .
$$

We have

$$
\alpha_{p, i}^{*}=\frac{H_{n}}{p}\left(i+p-\frac{1}{\frac{1}{i}+\ldots \frac{1}{i+p}}\right),
$$

which is an increasing function of $i$. It follows that

$$
\min _{i=1, \ldots, n-p} \alpha_{p, i}^{*}=\alpha_{p, 1}^{*}=H_{n}\left(1+\frac{1}{p}-\frac{1}{p H_{p+1}}\right) .
$$

Using (3.18) and (3.25), we calculate

$$
\alpha_{2 p}=\frac{H_{n}}{1+\frac{1}{\gamma}}\left(1+\frac{1}{p}\right), p=1, \ldots, n-1,
$$

which is a decreasing function of $p$. Observing that $\alpha_{p, 1}^{*}$ defined by (3.28) is also a decreasing function of $p$, we have $\alpha^{*}=\min \left(\alpha_{2(n-1)}, \alpha_{n-1,1}^{*}\right)$. Using $\gamma \leq n H_{n}-1$, we have $\alpha^{*}=\alpha_{2(n-1)}$ and we obtain (3.26). Using (3.18) and (3.12), we obtain (3.27). At the critical value $\alpha_{c}=H_{n}$, a degenerate $n$-fold bifurcation of the speed curve occurs: for $\alpha<\alpha_{c}$ the speed equation (3.2) has no solution. At $\alpha=\alpha_{c}$ a family of $n$ branching points $\left(H_{n}, c_{p}^{l}\right), p=1, \ldots, n$, is defined, in the $\alpha$-c plane, which gives, as 


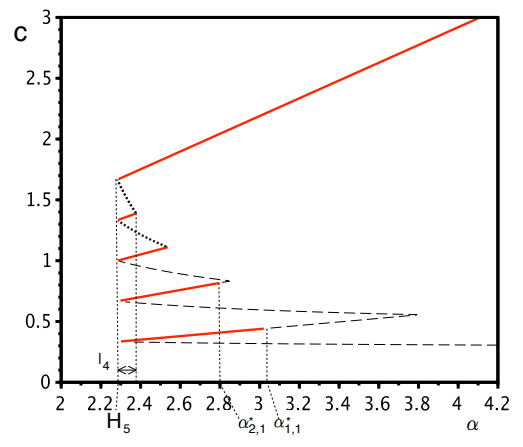

(a)
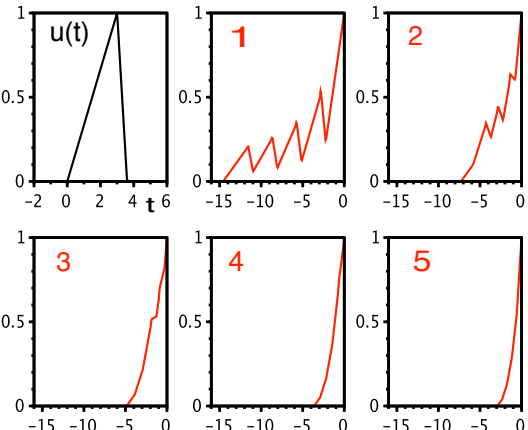

(b)

FIG. 3.3. Traveling signals in a transmission line where $w_{i}=1 / i$ (nonnormalized), $n=5$, and $\gamma=5\left(\tau_{1}=3, \tau_{2}=0.6\right)$. (a) Speed curve as a function of $\alpha$. The red line denotes stable propagating signals and long-dashed lines are for nonadmissible solutions. The five distinct red branches pertain to the different $p$-signals where $p=1, \ldots, 5$ from bottom $(p=1)$ to top $(p=5)$ respectively. The critical values $\alpha_{p, 1}^{*}$ above which p-signals do not satisfy the solvability condition, are indicated. Parts of the branches of the speed curve are not admissible and we can observe that, for $p=1$ and $p=2$, the critical value $\alpha_{p, 1}^{*}$ is lower than the upper bound of the corresponding $\alpha$-interval where p-signals are defined. Interval $I_{4}$ consits of $\alpha$-values where 4-signals exist and corresponds to the region of multistability between the five p-signals. (b) Plots of the coupling function $u(t)$ and the five p-signal profiles, labeled $p=1, \ldots, 5$, in the travelling wave coordinate $\xi=n-c_{p} t$, where $c_{p}$ is the velocity of the p-signal.

$\alpha$ increases, $2 n$ branches of solutions that are alternately associated with an unstable signal and a $p$-wave. In Fig. 3.3, the multistability between $n$ distinct signals is illustrated for $n=5$ and for parameters satisfying conditions of Theorem 3.1. The left turning points of the speed curve are vertically aligned, and a nonempty $\alpha$-interval can be found for which the $n$ different $p$-signals, $p \in\{1, \ldots, n\}$, can propagate stably along the transmission line. For large $n$, we have $H_{n} \sim \ln n$, and for $\gamma=O(n \ln n)$ (such that $\gamma<n \ln n-1$ ), we have $\left|I_{n-1}\right|=O(\ln n / n)$, and the region of coexistence of $n$ traveling signals shrinks as $n$ increases.

For $\gamma>n H_{n}-1$, one has $\alpha^{*}=\alpha_{n-1,1}^{*}$ and the interval of multistability is reduced to $] H_{n},\left(n H_{n}-1\right) /(n-1)[$.

For $n=2$, we have $\left(w_{1}, w_{2}\right)=(2 / 3,1 / 3)$ and application of Theorem 3.1 implies, for $1<\gamma \leq 2$, the stable propagation of regular signals with two different velocities $c_{1}=2 \alpha /\left(3 \tau_{1}\right)$ and $c_{2}=2 c_{1}$ when $\left.\alpha \in\right] 3 / 2,3 /\left(1+\gamma^{-1}\right)[$. A comprehensive analysis of second-neighbor coupling chains is done in section 4 and the maximal domain, in the parameter space, of signal coexistence is determined analytically.

Another specific configuration of the transmission line is obtained when $\alpha_{2 p}$ values $(p=1, \ldots n-1)$ are identical (vertical alignment of the right turning points of the speed curve). Calculations lead to the following network connectivity:

$$
w_{1}=\frac{1}{\kappa}, \quad \text { and } w_{i}=\frac{1}{2 i \kappa}, \quad i=2, \ldots, n,
$$


where $\kappa=\left(1+H_{n}\right) / 2$ is the normalization coefficient. In this case, the bounds of $D_{p}$, the $\alpha$-interval where $p$-signals are defined, are given by

$$
\alpha_{2 p-1}=\frac{2 p \kappa}{p+1}, \quad \text { and } \quad \alpha_{2 p}=\frac{2 \kappa}{1+\frac{1}{\gamma}} .
$$

The critical value $\alpha_{p, i}^{*}$ increases with $i$ and $\min _{i=1, \ldots, n-p} \alpha_{p, i}^{*}=\alpha_{p, 1}^{*}$ which is an increasing function of $p$. It follows that $\min _{p, i} \alpha_{p, i}^{*}=\alpha_{1,1}^{*}=4 / 5\left(1+H_{n}\right)$. Observing that $\left(\alpha_{2 p-1}\right)$ is a strictly increasing sequence, one may ensure the existence of an $\alpha$-interval of multistability between the $p$-signals where $p=1, \ldots, n$, when

$$
\min \left(4 / 5\left(1+H_{n}\right),\left(1+H_{n}\right) /\left(1+\gamma^{-1}\right)\right)>\alpha_{2 n-1} .
$$

For $\gamma \leq 4$, the inequality is fulfilled when $\gamma>n$ and, for $\gamma>4$, the inequality gives $n<4$. Thus, for a network connectivity given by (3.30), the parameter values for which $p$-signals exist simultaneously $(p=1, . ., n)$ are $n \in\{2,3\}$ and $\gamma>n$. For $n=2$, the $\alpha$-interval of multistability between 1 -signal and 2 -signal is $] 5 / 3,5 /(2(1+$ $\left.\gamma^{-1}\right)$ ) [ when $2<\gamma \leq 4$, and ]5/3,2[ when $\gamma>4$. For $n=3$, this interval becomes ] $17 / 8,17 /\left(6\left(1+\gamma^{-1}\right)\right)[$ when $3<\gamma \leq 4$ and $] 17 / 8,34 / 15[$ when $\gamma>4$.

The vertical alignment of the right turning points reflects a $(n-1)$-fold degeneracy of the speed curve, similar to the one observed with the left-hand alignment previously discussed. For a connectivity of the transmission line given by (3.30), the speed equation has $n-1$ branching points that give rise to $n-1$ branches associated with $p$-signals, $p=1, \ldots, n-1$. The fast-transmission is also a solution and we have, potentially, $n$ different waves. However, unlike the left alignment, the existence of traveling signals is restricted by the solvability condition and the coexistence of $n$ distinct signals can hardly be achieved. As $n$ increases, the intersection of the $\alpha$ intervals of existence and admissibility of signals shrinks and the multistability is only obtained at small $n$ values (the trivial case $n=2$, and third-neighbor interactions $n=3)$.

4. Second-neighbor interactions. We consider here the case where $n=2$, which is a chain where a node is connected to its nearest neighbor and to its next nearest neighbor with connection weights $w_{1}$ and $w_{2}$, respectively.

Let us first examine the particular case where $w_{2}=0$ that corresponds to nearestneighbor coupling, i.e., $n=1$. The excitable chain mimics the behavior of a row of dominoes where a slight push of the first domino causes it to topple until it strikes the second and, in turn, hits the next one. A sequence of events is generated that takes the form of a traveling signal with upright dominoes in front and toppled dominoes behind wave passage. In the nearest-neighbor coupling transmission line, propagation of signal occurs only if an element is able to activate its successor, which gives $\alpha \geq 1$ (similar to the constraint on the ratio between the spacing of the dominoes and the height of the dominoes). The signal is unique and its velocity is given by $c=\alpha / \tau_{1}$ that can be interpreted as the ratio between the spacing between dominoes and the free fall time of the dominoes. In this regime, the decaying phase of the coupling function $u$ is irrelevant. The characteristic polynomial associated with the soliton is $P(\lambda)=w_{1} / \tau_{1}(\lambda-1)$ and the wave is stable. The critical case $w_{2}=1\left(w_{1}=0\right)$ represents two nonconnected chains, where the dynamics of each chain is similar to the one described above (for $w_{2}=0$ ).

For $n=2$, the existence of a traveling wave is related to the existence of a solution $c$ of

$$
\alpha w_{1} u(1 / c)+\alpha w_{2} u(2 / c)=1,
$$


together with the solvability criterion

$$
\alpha w_{1} u(\xi+1 / c)+\alpha w_{2} u(\xi+2 / c)<1, \forall \xi<0 .
$$

The stability of the wave is determined by the eigenvalue

$$
\lambda=-\frac{w_{2} u^{\prime}(2 / c)}{w_{1} u^{\prime}(1 / c)+w_{2} u^{\prime}(2 / c)} .
$$

The connectivity of the transmission line, described by $w_{1}$ and $w_{2}$ such that $\left|w_{1}\right|+$ $\left|w_{2}\right|=1$, can be controlled using a single parameter $0<w<1$ leading to four different network architectures $\left(w_{1}, w_{2}\right)$ given by: (E) $(w, 1-w)$ purely excitatory, (EI) $(w, w-1)$ excitatory-inhibitory, (IE) $(-w, 1-w)$ inhibitory-excitatory, and (I) $(-w, w-1)$ purely inhibitory. Purely inhibitory networks do not allow the propagation of signals since the positive threshold of excitation cannot be reached. The detailed study of the three transmission lines (E), (EI) and (IE) is presented in Appendix A. The existence and stability of traveling signals is analytically determined and the characteristic speed curves are given as parameters changed. Depending on $\gamma$, we distinguish among three cases. For $\gamma>1,1$-signals exist and may coexist with 2signals. When $\gamma<1$, multistability is not possible. The case $\tau_{1}=\tau_{2}(\gamma=1)$ is also briefly discussed.

The locus of existence and stability of traveling signals in the $(w, \alpha)$ plane is shown in Figure 4.1(a) for $\gamma>1$. Three different signals may propagate stably: a 2 -signal referred to as a fast wave, a (1,1)-signal and a 1-signal. One may observe a region of bistability between 2-signals and 1-signals. This is illustrated in Fig. 4.1(b) where the same transmission line supports the propagation of two signals with different waveforms and distinct velocities. From the analysis presented in Appendix A, we define the two critical coupling weights

$$
w_{a}=\frac{\gamma}{\gamma+1} \text { and } w_{b}=\frac{2 \gamma}{2 \gamma+1}
$$

and we stress the following properties of signal propagations in second-neighbor coupling networks:

- Only excitatory (E) and excitatory-inhibitory (EI) transmission lines support the stable propagation of simple waves. There exists a nonzero minimal wave speed below which no regular signal propagates. This minimum is greater than $\tau_{1}^{-1}$ and is associated with the minimal $\alpha$-value allowing stable propagation, except in the bistability case.

- Bistability, i.e. the coexistence of two stable signals, occurs only in excitatory networks. Necessary and sufficient conditions are

$$
\begin{aligned}
& \gamma>1, \frac{1}{2}<w<w_{b}, \\
& \text { and } \max \left(\frac{1}{w}, \frac{2}{2-w}\right)<\alpha<2 \min \left(1, \frac{w_{a}}{w}\right) .
\end{aligned}
$$

Under these conditions, signals that propagate along the transmission line may have two different velocities: $c=\alpha(2-w) / \tau_{1}$ or $c=\alpha w / \tau_{1}$. When $w<w_{a}$ the profile of the slowest signal is nonincreasing.

Different bifurcation scenarios lead to multistability of signals. Let us fix the two parameters $\gamma>1$ and $w \in] 1 / 2, w_{b}[$, and consider $\alpha$ as the bifurcation 


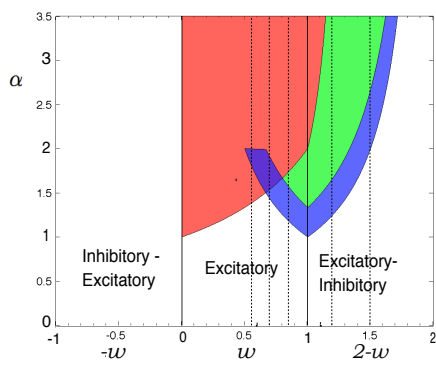

(a)
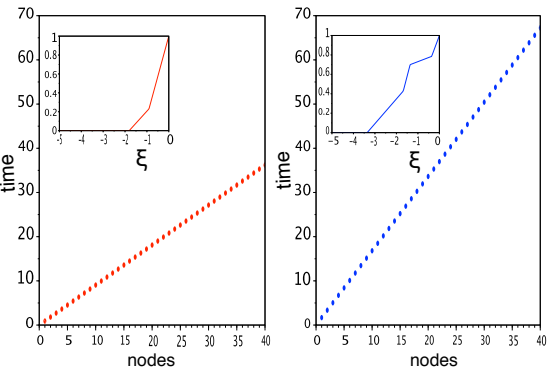

(b)

FIG. 4.1. (a) Locus of existence of stable signals for $n=2$ and $\gamma>1$ in the $(w, \alpha)$ parameter plane where the weight parameter is shifted in order to describe the three different transmission lines; inhibitory-excitatory, purely excitatory, and excitatory-inhibitory (from left to right, respectively). Fast waves (2-signals) are in red. The blue region denotes 1 -signals and the green area is associated with $(1,1)$-signals. Boundaries of the different regions are analytically determined (see Appendix A). The speed diagrams (wave speed, $c$, with respect to the global coupling strength, $\alpha$ ) corresponding to the $w$-values marked by vertical dotted lines are plotted in Appendix A. For excitatory network $(w \in(0,1))$, there exists a region where 2-signals and 1-signals coexist. Panel (b) shows the raster plots (emitting times) of network activity for parameters in the bistability region $(w=0.7$ and $\alpha=1.7)$ using two different initial stimulations. The left plot shows the fast propagation of the 2signal (in red) while in the right plot the 1-signal propagates. The two insets show the corresponding signal profiles. Plots are done for $\gamma=2\left(\tau_{1}=2, \tau_{2}=1\right)$.

parameter. As $\alpha$ increases, branching bifurcations appear at $\alpha=1 / w$ and $\alpha=2 /(2-w)$, which are associated with the 1-signal and the fast-signal, respectively. These two branching points are reached simultaneously when $w=2 / 3$, a case that has been reported previously as the vertical alignment of the left branching points. Another branching bifurcation occurs at $\alpha=2 w_{a} / w$ and is associated with the 1-signal (as $\alpha$ decreases). However, when $w<w_{a}$, this bifurcation leads to a nonadmissible signal and a boundary bifurcation occurs at $\alpha=2$, where the 1 -signal satisfies the solvability condition (as $\alpha$ decreases). One may observe that a necessary condition for multistability is $3 / 2<\alpha<2$.

If $\gamma$ is used as the bifurcation parameter, the bifurcation equation $\gamma=1$ is associated with a qualitative change of signal classes. When $\gamma>1$, the 1 signal class exists, allowing multistability, whereas for $\gamma<1$ the $(0,2)$-signal class appears but does not contain admissible signals.

The weight $w$ can also be used as a bifurcation parameter (see the brief discussion given in Appendix A).

- When $w \geq w_{b}$, there is a continuum, i.e., without gap, of wave speed values (associated with stable signals) as $\alpha$ changes from the minimal wave speed to a maximal value. The maximal value is unbounded as $\alpha$ increases except in excitatory-inhibitory networks where the wave speed is bounded when $w \leq$ $2 / 3$.

- Set-valued speed curves. A degenerate situation occurs in an excitatory network when $w=w_{b}$ where, for $\alpha=(1+2 \gamma) /(1+\gamma)$, the wave speed may take any value in $\left[2 /\left(\tau_{1}+\tau_{2}\right), 2 / \tau_{1}\right]$ when $\gamma \geq 1$, or $\left[1 / \tau_{1}, 2 / \tau_{1}\right]$ when $\gamma<1$. The 
associated eigenvalue is $\lambda=1$ and the corresponding signal is stable (but not asymptotically stable).

For identical time constants $(\gamma=1)$, the coupling function is a rescaled triangle function. In this case we have $w_{a}=1 / 2$ and $w_{b}=2 / 3$ and the region where 1 -signals exist disappears and a direct transition from a $(0,1)$-signal to a $(1,1)$-signal is observed as $c$ increases. In particular, the region of bistability no longer exists.

4.1. Third-neighbor interactions. We have shown the existence of a parameter regime allowing multistability between $n$ traveling signals, for an arbitrary $n$. The exact domain of multistability has been determined entirely for $n=2$. In this section, we determine geometrically, for $n=3$, the connectivity of the transmission line allowing the propagation of distinct signals.

We direct our attention to positive weights and assume $\gamma>2$ since our analysis above shows that this is the parameter regime where multistability is possible. In the parameter plane $\left(w_{1}, w_{2}\right)$, we determine the locus of coexistence of the three stable traveling waves: 3-signals, 2-signals and 1-signals. The analysis is detailed in Appendix B and the results are illustrated in Figure 4.2. where the active constraints are shown (labeled lines) and the resulting domain of multistability between three signals is depicted. Speed curves shown in Fig.4.2 illustrate some characteristic regimes: multistability between the three $p$-signals $(p=1,2,3)$ (speed curve for $\left.\left(w_{1}, w_{2}\right)=(0.65,0.15)\right)$; multistability between three signals is not possible because one of the $p$-signals is not admissible (for $\left.\left(w_{1}, w_{2}\right)=(0.43,0.27)\right)$ or because a folding condition is not satisfied (for $\left(w_{1}, w_{2}\right)=(0.6,0.3)$ or $(0.7,0.05)$ ). We note the bistability between two propagating signals in the depicted speed curves.

5. Discussion. Many efforts have been made in the past to use excitable media as computational systems (see [24] and references therein). An axiomatic theory of excitable media has been developed where the cooperative effect generated by many simple identical components is exploited to achieve computational tasks [44]. Due to their threshold behavior, cellular automaton models are particularly suitable for excitable media description [21, 16], but they fail to capture continuous-time-dependent properties. In contrast, detailed models of excitable media describe the fine temporal structure of the dynamics but their study is difficult beyond numerical simulations. Solvable models are desirable to derive valuable insights into the dynamics of nonlinear waves. In this paper, we formulate a phenomenological model of excitable media consisting of a continuous integration of incoming inputs supplemented with threshold. Our model has a dynamical structure combined with a spatial organization while being exactly solvable. Unlike previous studies on lattice automaton-type models, we do not use mean field approximations but explore the fine temporal dynamics of the system.

Solitary waves that switch the medium from the resting state to another stationary solution are ubiquitous in excitable media. These switching waves establish a serial (or avalanche-type) communication bridge between two distant nodes and allow the fast and robust transmission of (one-bit) warning messages. Conveying several bits in one transmission can hardly be achieved in numerous natural systems, especially when the state cannot be restored immediately after the first excitation. We have shown here that for a one-dimensional information channel with $n$ th-neighbor interactions, an $n$-fold degeneracy of the speed curve induces the coexistence of $2 n$ propagating signals, $n$ of which are stable, and allows transmission of $n$ distinct symbols. This configuration is obtained in purely excitatory networks with appropriate connection 


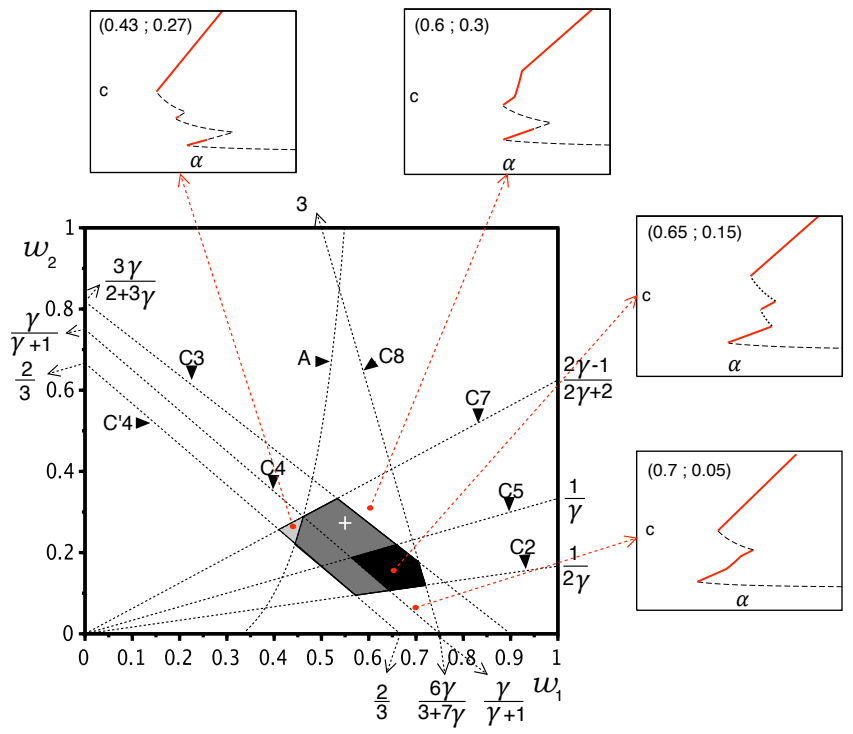

FIG. 4.2. Multistability in excitable transmission lines with third-neighbor interactions $(n=3)$. We take $\gamma>2$ (plot is done for $\gamma=3$ ). The gray and black regions define parameters $\left(w_{1}, w_{2}\right)$ where multistability is possible, i.e. , there exists a nonempty parameter set such that the three p-signals $(p=1,2,3)$ propagate in the chain. In the black region, sufficient conditions of multistability are satisfied where the entire $\alpha$-interval of p-signal existence is admissible for each p-signal. In the light gray region, it is possible to find each of the three p-signals but they fail to exist simultaneously (due to the nonadmissibilty of one of them). Boundaries (given by the labeled curves) between the different regions are determined analytically in Appendix $B$ and some characteristic points are provided here. Only the active constraints have been represented. The white cross corresponds to the conditions of theorem $3.1\left(w_{1} \approx 0.54\right.$ and $\left.w_{2} \approx 0.27\right)$. Speed curves (velocity $c$ with respect to the coupling $\alpha)$ for connectivity parameters, $\left(w_{1}, w_{2}\right)$, located at the red points are depicted. The solid red lines denote stable signals. The dotted lines represent unstable solutions and the dashed lines are for nonadmissible solutions (i.e., candidate solutions that violate the solvability condition).

weights and for a time constants ratio greater than $n-1$.

Two distinct spreading activities in unidimensional excitable media have already been observed $[23,14,3]$. They are characterized by two separate propagation velocities and two different waveforms. This bistability comes from the existence of two different excitability thresholds that induce the propagation of small-amplitude and highamplitude traveling waves. The coexistence of fast and slow pulses in excitable neural media has already been pointed out in integrate-and-fire-type networks $[12,33]$ and in neural field models [7] but the slow pulses turn out to be unstable [5]. Further analysis has shown that bistability can be obtained in two-layer one-dimensional lattices $[18,19]$. In many studies, the admissibility (or solvability) criterion for traveling wave candidates is not discussed, making unclear the effective existence of two signals. Our analysis suggests that multistability can be obtained in homogeneous networks with extended spatial connections and does not require the existence of multiple thresholds.

One may wonder whether multistability of traveling signals is peculiar to the system introduced here or if it is a general feature of lattice excitable systems. In order to corroborate our analysis, let us consider the Connor-Stevens model for action potential 


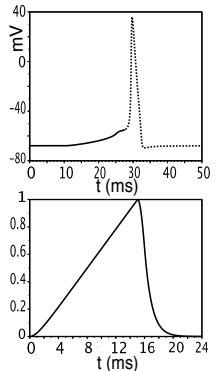

(a)

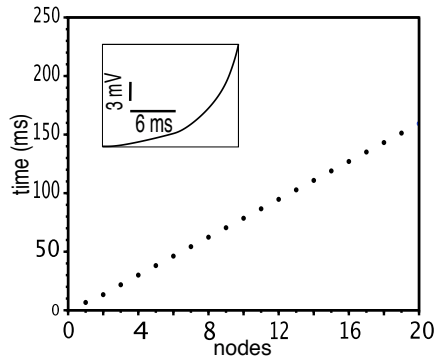

(b)

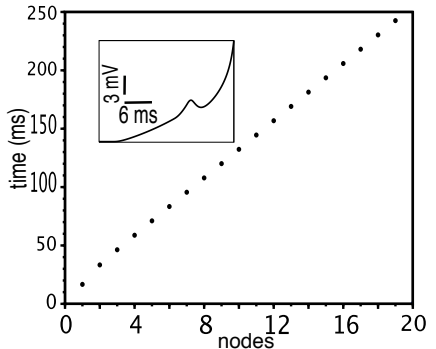

(c)

FIG. 5.1. Multistability in a chain of excitable cells of Connor-Stevens type. Panel (a)-top shows the membrane potential of the Connor-Stevens neuron after the injection of the input current shown below (applied at $t=20 \mathrm{~ms}$ ). The dotted line indicates the stereotyped spike response of the model after the threshold is reached $\left(a t v_{t h}=-55 \mathrm{mV}\right)$ and the solid line describes the subthreshold dynamics before spike emitting. In (b)-(c), rastergrams obtained from the numerical integration of a chain of synaptically coupled Connor-Stevens neurons. Each cell in the chain has $n=2$ presynaptic neurons and an incoming spike induces a depolarization of the membrane potential of the postsynaptic neurons that is described by the excitatory postsynaptic current shown in panel (a) (bottom). Two distinct initial stimulations (using an injected current into the first nodes of the chain) generate the propagation of two different signals with subthreshold waveprofiles shown in the respective insets. (b) Fast wave with an increasing waveform and a velocity $c \sim 0.15 \mathrm{~ms}^{-1}$ and (c) 1-signal with a nonmonotonic profile and a velocity $c \sim 0.06 \mathrm{~ms}^{-1}$.

generation (for references and equations, see [8]). It is a detailed conductance-based neuron model that displays a type-I excitability where a pulse, without damping oscillations (unlike the classical Hodgkin-Huxley model), is generated after a brief subthreshold input current, as shown in Fig. 5.1 (a) (top). The numerical simulation of a Connor-Stevens transmission line with second-neighbor synaptic coupling shows the simultaneous existence of two traveling signals (see Fig. 5.1 (b),(c)). Further investigations are necessary to probe multistability for larger interactions and for other detailed excitable models.

We have focused on simple waves, already reported as monochromatic waves, 1lurcher, or smooth waves, where the dynamics at each lattice point follows the same trajectory. Localized waves with a spatiotemporal periodicity have been recently analyzed in excitable lattices $[25,39]$ and numerical simulations have indicated the existence of discontinuous pulses that propagate in a saltatory fashion along excitable neural systems $[20,35,18]$. These nonclassical signal propagations can coexist with simple traveling waves [41] and thus participate in the communication capabilities of excitable transmission lines [14].

Appendix A. Second-neighbor interactions. We present here a comprehensive study of regular signals in the case $n=2$. Equations are the following:

$$
\begin{aligned}
& \alpha w_{1} u\left(c^{-1}\right)+\alpha w_{2} u\left(2 c^{-1}\right)=1, \\
& \alpha w_{1} u\left(\xi+c^{-1}\right)+\alpha w_{2} u\left(\xi+2 c^{-1}\right)<1, \forall \xi<0, \\
& w_{1} u^{\prime}\left(c^{-1}\right)+w_{2} u^{\prime}\left(2 c^{-1}\right) \geq 0, \\
& \lambda=-\left(1+\frac{w_{1} u^{\prime}\left(c^{-1}\right)}{w_{2} u^{\prime}\left(2 c^{-1}\right)}\right)^{-1},
\end{aligned}
$$


which give, respectively, the velocity $c$ of candidate solutions, the admissibility criterion, a necessary condition of existence, and the eigenvalue associated with the signal stability. The analysis is based on the location of the two time events $t_{1}=c^{-1}$ and $t_{2}=2 c^{-1}$ with respect to $\tau_{1}$ and $\tau_{1}+\tau_{2}$. We distinguish between the following wave types:

- 2-signal. This case has been reported as the fast-transmission regime that is obtained when $c>2 / \tau_{1}$. From (5.1) the wave speed is given by

$$
c=\frac{\alpha\left(w_{1}+2 w_{2}\right)}{\tau_{1}}
$$

and the existence of fast waves requires $\alpha>2 /\left(w_{1}+2 w_{2}\right)$. The necessary condition (5.3) rewrites $w_{1}+w_{2} \geq 0$. The stability is determined by $\lambda=$ $-w_{2} /\left(w_{1}+w_{2}\right)$ and, consequently, the fast wave is stable if and only if $w_{1} \geq$ $\max \left(0,-2 w_{2}\right)$.

- $(\mathbf{1}, \mathbf{1})$-signal. This case is obtained for intermediate speed values such that $\max \left(1 / \tau_{1}, 2 /\left(\tau_{1}+\tau_{2}\right)\right)<c<2 / \tau_{1}$. The wave speed is given by

$$
c=\frac{w_{1}-2 \gamma w_{2}}{\tau_{1}\left(\frac{1}{\alpha}-w_{2}(1+\gamma)\right)} .
$$

The necessary condition (5.3) reads as $w_{1} \geq \gamma w_{2}$ and the stability is determined by $\lambda=\gamma w_{2} /\left(w_{1}-\gamma w_{2}\right)$. The $(1,1)$-signal is stable if and only if $w_{1} \geq \max \left(0,2 \gamma w_{2}\right)$.

- 1-signal. For moderate speed values defined by $1 / \tau_{1}<c<2 /\left(\tau_{1}+\tau_{2}\right)$ the wave speed is given by

$$
c=\frac{\alpha w_{1}}{\tau_{1}},
$$

and the signal is asymptotically stable $(\lambda=0)$. Such waves exist only if $\gamma>1$.

- (0,2)-signal. At small speed values, $2 /\left(\tau_{1}+\tau_{2}\right)<c<1 / \tau_{1}$, it is possible to find network architecture that supports $(0,2)$-signals. In this case, the wave speed is given by

$$
c=\frac{w_{1}+2 w_{2}}{\tau_{2}\left((1+\gamma)\left(w_{1}+w_{2}\right)-\frac{1}{\alpha}\right)}
$$

and the stability is determined by $\lambda=-w_{2} /\left(w_{1}+w_{2}\right)$. The existence of such waves requires $\gamma<1$.

- (0,1)-signal. These signals are not admissible.

We now distinguish between the sign of the connection weights $\left(w_{1}, w_{2}\right)$. Let $0<$ $w<1$ be the connectivity parameter. We identify three different network architectures: $(w, 1-w)$ excitatory, $(w, w-1)$ excitatory-inhibitory, and $(-w, 1-w)$ inhibitory-excitatory. For each architecture, it is possible to clarify the condition for the existence of a $c$-solution of (5.1) and the solvability condition (5.2).

Excitatory chain. The fast wave exists for $\alpha>2 /(2-w)$. The waveprofile $X(\xi)$ is strictly increasing and the signal is asymptotically stable $(\lambda=w-1)$.

Let us consider the two critical weight parameters, $w_{a}$ and $w_{b}$ given by (4.4). The $(1,1)$-signal exists if and only if $w>w_{a}$ and $\left.\alpha \in\right] 2 w_{a} / w, 2 /(2-w)\left[\right.$ when $w>w_{b}$, 
or $\alpha \in] 2 /(2-w), 2 w_{a} / w\left[\right.$ when $w<w_{b}$. The $(1,1)$-signal trajectory is increasing and asymptotically stable if and only if $w>w_{b}$. On the interval where $(1,1)$-signals exist, the speed curve $c(\alpha)$ is strictly increasing if and only if $w>w_{b}$. For $w=w_{b}$, the speed curve, in the $(\alpha, c)$ plane, is a vertical segment at $\alpha=2 w_{a} / w_{b}$ and the associated waves are stable.

For $\gamma>1,1$-signals exist if and only if $\alpha \in] 1 / w, 2 w_{a} / w\left[\right.$ when $w>w_{a}$ and $\left.\alpha \in\right] 1 / w, 2[$ otherwise. When $w>w_{a}$, the signal profile is strictly increasing. When $w<w_{a}$, the waveprofile has a local maximum at $\xi=\tau_{1}\left(1-2(\alpha w)^{-1}\right)$ given by $\alpha-1$.

For $\gamma=1$, the coupling function is the triangular function, and we have $w_{a}=1 / 2$ and $w_{b}=2 / 3$. The analysis remains unchanged for 2 -signals and $(1,1)$-signals whereas 1-signals no longer exist.

Results are presented in two distinct plots done for $\gamma>1$ and $\gamma<1$ (see Fig. 5.2(a), (b), respectively). In each panel, three different cases are depicted that show the characteristic speed diagrams in the $c-\alpha$ plane for $w<w_{a}, w_{a}<w<w_{b}$ and $w>w_{b}$.

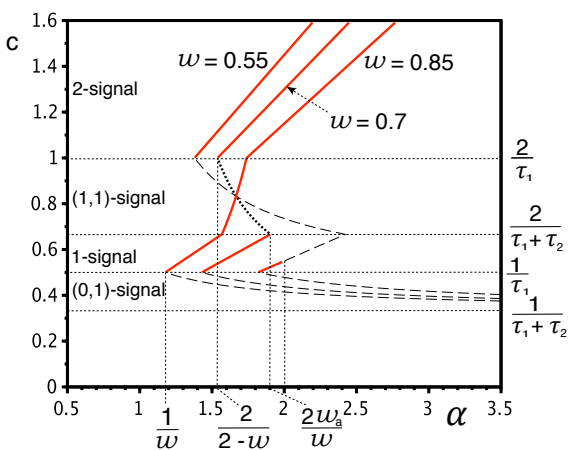

(a) $\gamma>1$

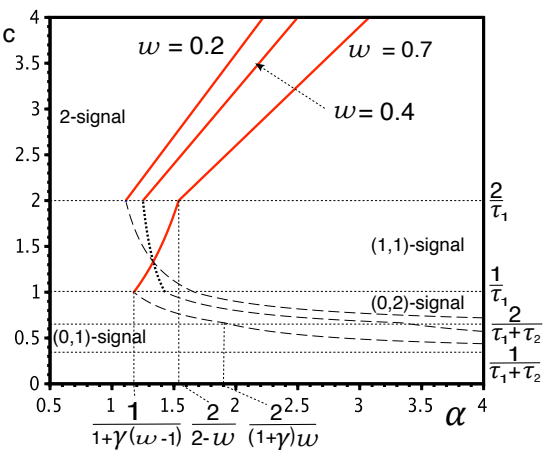

(b) $\gamma<1$

FIG. 5.2. Wave speed $c$ as a function of the global coupling strength $\alpha$ in a transmission line with excitatory second-neighbor interactions $(n=2)$. For each panel, we consider three different values of $w$ that illustrate qualitatively different configurations of the speed curve. Red curves denote stable signals, dotted curves are for unstable waves and long-dashed curves represent candidate solutions that are not admissible. Thin vertical dotted lines give characteristic $\alpha$-values of the curves and thin dotted horizontal lines are the c-values that define the boundaries between the different signal classes. (a) Plot of the speed curves for $\gamma>1$ (we take here $\tau_{1}=2$ and $\tau_{2}=1$ ) and for $w=0.55,0.7$ and 0.85 (we have here $w_{a}=2 / 3$ and $w_{b}=4 / 5$ ). One observes a bistability regime if $w<w_{b}$ and the nonadmissibility of the $(1,1)$-signal when $w<w_{a}$ and $\alpha>2$. (b) Plot for $\gamma<1$ (we take $\tau_{1}=1$ and $\tau_{2}=2$ ) and for $w=0.3,0.4$ and 0.7 . We have here $w_{a}=1 / 3$ and $w_{b}=1 / 2$. One may observe that for $w<w_{a}$, only 2-signals are admissible. The speed curve has a unique turning point at $c=2 / \tau_{1}$ for $w<w_{b}$ or at $c=1 / \tau_{1}$ for $w>w_{b}$.

For $\gamma>1$, the speed diagram in the $c$ - $w$ plane (not shown here) presents for $\alpha \in] 1,3 / 2[$ two nonconnected curves associated with the fast-signal and the 1-signal that are defined on two disjoint intervals, $w \in] 0,2-2 / \alpha$ and $w \in] 1 / \alpha, 1[$, respectively. As $\alpha$ increases and reaches $3 / 2$, there exists a $w$-range where both curves are superimposed (allowing multistability). For $\alpha=1+w_{a}$, the two curves connect at $w=w_{b}$ and finally, as $\alpha$ further increases, separate into two nonconnected curves where the lower curve represents nonadmissible solutions when $\alpha>2$. 
Excitatory-Inhibitory chain. The fast wave speed is given by $c=\alpha(3 w-2) / \tau_{1}$ and its domain of existence is $w>2 / 3$ and $\alpha>2 /(3 w-2)$. The fast wave is asymptotically stable $(\lambda=(1-w) /(2 w-1))$. The $(1,1)$-wave is asymptotically stable with a velocity that increases with $\alpha$. In order to clarify the domain of existence of $(1,1)$ signals and to analyze 1-signals and $(0,2)$-signals, we distinguish between different cases, depending on $\gamma$ and $w$.

Let us assume $\gamma \geq 1$. The 1 -signal is stable and exists for $\alpha \in] 1 / w, 2 w_{a} / w[$. The $(1,1)$-signal is stable and exists for $\alpha \in] 2 w_{a} / w, 2 /(3 w-2)[$ when $w>2 / 3$ and for $\alpha \in] 2 w_{a} / w,+\infty[$ when $w \leq 2 / 3$.

For $\gamma<1$, the $(1,1)$-signal is stable and exists only when $w>(1-\gamma) /(2-\gamma)$. For $w>2 / 3$, the $(1,1)$-signal exists if and only if $\alpha \in](\gamma(1-w)+2 w-1)^{-1}, 2 /(3 w-2)[$. For $(1-\gamma) /(2-\gamma)<w \leq 2 / 3$, the interval of existence becomes $\alpha \in](\gamma(1-w)+$ $2 w-1)^{-1},+\infty[$. The $(0,2)$-signal is unstable and its domain of existence is given by $\alpha \in] 2 /(w(1+\gamma)),(\gamma(1-w)+2 w-1)^{-1}[$ for $(1-\gamma) /(2-\gamma)<w<1 / 2$ and ] $2 /(w(1+\gamma)),+\infty[$ for $w \leq(1-\gamma) /(2-\gamma)$.

Results in the $(w, \alpha)$ parameter plane are summarized in Figure 5.3 for the different transmission lines.

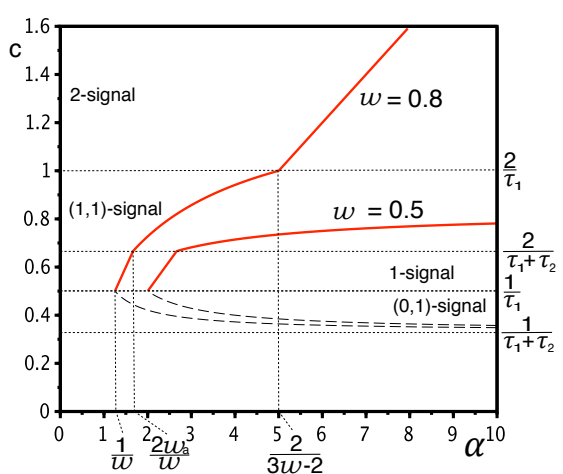

(a) $\gamma>1$

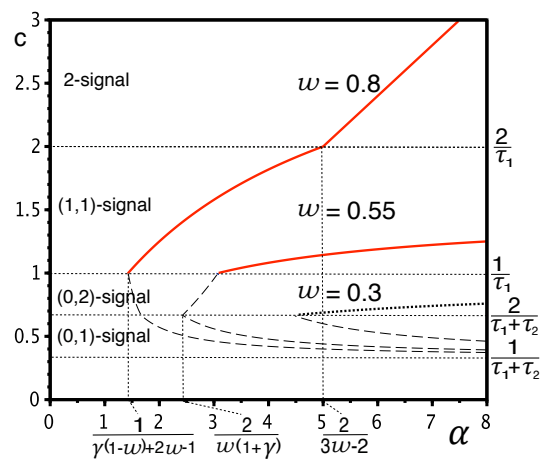

(b) $\gamma<1$

FIG. 5.3. Wave speed $c$ as a function of $\alpha$ in an excitatory-inhibitory transmission line with second-neighbor interactions $(n=2)$. Same legend as in Fig. 5.2. (a) Plot of the speed curves for $\gamma>1$ (we take here $\tau_{1}=2$ and $\tau_{2}=1$ ) and for $w=0.8$ and 0.5 that illustrate the two cases $w>2 / 3$ and $w<2 / 3$. (b) Plot for $\gamma<1$ (we take here $\tau_{1}=1$ and $\tau_{2}=2$ ) and for $w=0.8,0.55$ and 0.3 . We have here $(1-\gamma) /(2-\gamma)=1 / 3$.

Inhibitory-Excitatory chain. We easily show that only fast waves exist for $w<0.5$ and the wave speed is given by $c=\alpha(2-3 w) / \tau_{1}$. The wave is unstable. Other wave types are not admissible.

Results, for $\gamma>1$, are illustrated in Fig. 4.1.

Appendix B. Multistability in transmission lines with third-neighbor interactions. We determine the regime allowing the coexistence of stable waves when parameters $\gamma, \alpha$ and $\left(w_{i}\right)_{i=1,2,3}$ are fixed. We restrict our attention to the case where $\gamma>2$. In the $(\alpha, c)$ plane, the speed curve is made up of six connected branches 
associated with six different classes of signals. Three branches are associated with the different $p$-signals $(p=1,2,3)$ and multistability is obtained when a nonempty intersection can be found between the related $\alpha$-intervals. In the following we derive conditions on network parameters allowing multistability between 1-signals, 2-signals and 3-signals.

Normalization gives $w_{1}+w_{2}+w_{3}=1$, and we obtain the condition

$$
\text { (C1) } w_{2}<1-w_{1} \text {. }
$$

The folding condition (3.19) for 1-signals gives

$$
\text { (C2) } w_{2}>\frac{1}{2 \gamma} w_{1}
$$

and, for 2-signals,

$$
\text { (C3) } w_{2}<\frac{3 \gamma}{2+3 \gamma}-\frac{1+3 \gamma}{2+3 \gamma} w_{1}
$$

A sufficient condition for solvability (stronger than (3.23)) is $\alpha_{p, i}^{*}>\alpha_{2 p}$, i.e., we require that each $p$-signal is an admissible solution on its entire interval of definition ]$\alpha_{2 p-1}, \alpha_{2 p}[$. This gives

$$
\text { (C4) } w_{2}>\frac{\gamma}{\gamma+1}-w_{1}
$$

for 2-signals. For 1-signals, we obtain

$$
\text { (C5) } w_{2}<\frac{1}{\gamma} w_{1}
$$

and

$$
\text { (C6) } w_{2}>-\frac{1+3 \gamma}{1+\gamma} w_{1}+\frac{2 \gamma}{1+\gamma}
$$

Finally, in order to find a nonempty intersection of the three intervals $] \alpha_{1}, \alpha_{2}[,] \alpha_{3}, \alpha_{4}[$, and $] \alpha_{5},+\infty\left[\right.$, we require $\alpha_{4}>\alpha_{1}$ that gives

$$
\text { (C7) } w_{2}<\frac{2 \gamma-1}{2(\gamma+1)} w_{1}
$$

and $\alpha_{2}>\alpha_{5}$ that reads

$$
\text { (C8) } w_{2}<3-\frac{3+7 \gamma}{2 \gamma} w_{1}
$$

Conditions $(\mathrm{C} 1)-(\mathrm{C} 7)$ are sufficient to guarantee the multistability (black region in Fig. 4.2). Necessary conditions are $\alpha_{p, i}^{*}>\alpha_{2 p-1}$ and conditions C4-6 can be weakened as follows:

$$
\left(C^{\prime} 4\right) w_{2}>\frac{2}{3}-w_{1},\left(C^{\prime} 5\right) w_{2}<w_{1},\left(C^{\prime} 6\right) w_{2}>1-2 w_{1},
$$

which are illustrated in Fig. 4.2 by the gray region together with the light gray zone. Finally, necessary and sufficient conditions for the existence of an $\alpha$-interval of multistability are (C1)-(C3), (C'4)-(C'6), together with the conditions $: \min \left(\alpha_{4}, \alpha_{2,1}^{*}\right)>\alpha_{1}$, 
$\alpha_{2,1}^{*}>\alpha_{5}, \alpha_{3}<\min \left(\alpha_{1,1}^{*}, \alpha_{1,2}^{*}\right)$ and $\min \left(\alpha_{2}, \alpha_{1,1}^{*}, \alpha_{1,2}^{*}\right)>\alpha_{5}$ that we will not detail here. We found that the only active constraint is $\alpha_{2,1}^{*}>\alpha_{1}$ that gives the nonlinear inequality

$$
\text { (A) } 3 w_{1}^{2}-w_{1}+3 w_{1} w_{2}-2 w_{2}>0 \text {. }
$$

Inequality (A) excludes the light gray region depicted in Fig. 4.2 from the parameter set where multistability between three signals can be found because 1-signals and 2 -signals cannot exist simultaneously.

\section{REFERENCES}

[1] V. Acary, O. Bonnefon, And B. Brogliato, Nonsmooth Modeling and Simulation for Switched Circuits, Springer Verlag, 69, Lect. Notes in Electr. Eng. (2011).

[2] J. B. Ackman, T. J Burbridge, And M. C. Crair, Retinal waves coordinate patterned activity throughout the developing visual system, Nature, 490 (2012), pp. 219-225.

[3] E. Alvarez-Lacalle, and E. Moses, Slow and fast pulses in 1-D cultures of excitatory neurons, J. Comput. Neurosci., 26 (2009), pp. 475-493.

[4] P. Bak, C. TAng, and K. Wiesenfeld, Self-organized criticality: an explanation of $1 / \mathrm{f}$ noise, Phys. Rev. Lett. 59 (1987), pp. 381-384.

[5] P. C. Bressloff, Synaptically generated wave propagation in excitable neural media, Phys. Rev. Lett., 82 (1999), pp. 2979-2982.

[6] J. H. E. Cartwright, E. Hernández-Garcia, and O. Piro, Burridge-Knopoff models as elastic excitable media, Phys. Rev. Lett., 79 (1997), pp.527-530.

[7] S. Coombes, Waves, bumps, and patterns in neural field theories, Biol. Cybern. 93 (2005), pp. 91-108.

[8] P. Dayan, and L.F. Aвbott, Theoretical Neuroscience: Computational and Mathematical Modeling of Neural Systems, MIT Press, Cambridge, MA (2005).

[9] D. Dhar, Self-organized critical state of sandpile automaton models, Phys. Rev. Lett., 64 (1990), pp. 1613-1616.

[10] L. Edelstein-Keshet, J. Wartmough, and D. Grunbaum, Do travelling band solutions describe cohesive swarms? An investigation for migratory locusts, J. Math. Biol., 36 (1998), pp. 515-549.

[11] C. J. Efthimiou, And M. D. Johnson, Domino waves, SIAM Rev., 49 (2007), pp. 111-120.

[12] B. Ermentrout, The analysis of synaptically generated traveling waves, J. Comput. Neurosci., 5 (1998), pp. 191-208.

[13] I. Farkas, D. Helbing, And T. Vicsek, Mexican waves in an excitable medium, Nature, 419 (2002), pp. 131-132.

[14] O. Feinerman, M. Segal, and E. Moses, Signal propagation along unidimensional neuronal networks, J. Neurophysiol., 94 (2005), pp. 3406-3416.

[15] A. Gabrielov, Abelian avalanches and Tutte polynomials, Phys. A, 195 (1993), pp. 253-274.

[16] M. Gerhardt, H. Schuster, and J. J. Tyson, A cellular automaton model of excitable media including curvature and dispersion, Science, 247 (1990), pp. 1563-1566.

[17] W. Gerstner, And W. M. Kistler, Spiking Neuron Models: Single Neurons, Population, Plasticity, Cambridge University Press, Cambridge, UK (2002).

[18] D. Golomb, And G. B. Ermentrout, Continuous and lurching traveling pulses in neuronal networks with delay and spatially decaying connectivity, Proc. Natl. Acad. Sci. USA, 96 (1999), pp. 13480-13485.

[19] D. Golomb, And G. B. Ermentrout, Bistability in pulse propagation in networks of excitatory inhibitory populations, Phys. Rev. Lett., 86 (2001), pp. 4179-4182.

[20] D. Golomb, X. J. Wang, and J. Rinzel, Propagation of spindle waves in a thalamic slice model, J. Neurophysiol., 75 (1996), pp. 750-769.

[21] J. M. Greenberg, and S. P. Hastings, Spatial patterns for discrete models of diffusion in excitable media, SIAM J. Appl. Math., 34 (1978), pp. 515-523.

[22] A. V. M. Herz, AND J. J. HopfiELd, Earthquake cycles and neural reverberations: collective oscillations in systems with pulse-coupled threshold elements, Phys. Rev. Lett., 75 (1995), pp. $1222-1225$

[23] B. Hochner, AND M.E. SPIRA, Two distinct propagating regenerative potentials in a single ethanol-treated axon, Brain Res., 398 (1986), pp. 164-168.

[24] A. V. Holden, and J. V. Tucker and B. C. Thompson, Can excitable media be considered as computational systems?, Phys. D, 49 (1991), pp. 240-246. 
[25] H. J. Hupkes, L. Morelli, And P. Stehlík, Bichromatic travelling waves for lattice Nagumo equations, SIAM J. Appl. Dyn. Syst., 18 (2019), pp. 973-1014.

[26] M. A. P. Idiart, L. F. Аввотt, Propagation of excitation in neural network models, Netw. Comput. Neural Syst., 4 (1993), pp. 285-294.

[27] J. Keener, And J. Sneyd, Mathematical Physiology, Springer-Verlag, New York 1998.

[28] V. I. Krinsky, V. N. Biktashev, and I. R. Efimov, Autowave principles for parallel image processing, Phys. D 49 (1991), pp. 247-253.

[29] M. W. Mueller, And W. D. ARnett, Propagating star formation and irregular structure in spiral galaxies, Astrophys. J., 210 (1976), pp. 670-678.

[30] J. D. Murray, Mathematical Biology II: Spatial Models and Biomedical Applications, Springer 3rd edition (2003).

[31] V. Perez-Munuzuri, V. Perez-Villar, and L. O. Chua, Propagation failure in linear arrays of Chua's circuits, Internat. J. Bifur. Chaos Appl. Sci. Eng., 2 (1992), pp. 403-406.

[32] V. Perez-Munuzuri, V. Perez-Villar, and L. O. Chua, Autowaves for image processing on a two-dimensional CNN array of excitable nonlinear circuits: flat and wrinkled labyrinths, IEEE Trans. Circuits Syst. I, 40 (1993), pp. 174-181.

[33] R. Osan, AND B. ERmentrout, The evolution of synaptically generated waves in one- and two-dimensional domains, Phys. D, 163 (2002), pp. 217-235.

[34] J. R. Raney, N. Nadkarni, C. Daraio, D. M. Kochmann, J. A. Lewis, and K. Bertoldi, Stable propagation of mechanical signals in soft media using stored elastic energy, Proc. Natl. Acad. Sci. USA, 113 (2016), pp. 9722-9727.

[35] J. Rinzel, D. Terman, X.-J. Wang, and B. Ermentrout, Propagating activity patterns in large-scale inhibitory neuronal networks, Science, 279 (1998), pp. 1351-1355.

[36] L. S. Schulman, And P. E. Seiden, Percolation and galaxies, Science, 233 (1986), pp. 425-431.

[37] S. Thorpe, D. Fize, and C. Marlot, Speed of processing in the human visual system, Nature, 381 (1996), pp. 520-522.

[38] J. R. R. TolkiEn, The Lord of the Rings: 50th Anniversary Edition, Houghton Mifflin Harcourt, 2004, pp. 747-748.

[39] A. Tonnelier, Propagation of spike sequences in neural networks, SIAM J. Appl. Dyn. Syst., 9 (2010), pp. 1090-1118.

[40] V. A. Vasiliev and Yu. M. Romanovskit and D.S. Chernavskit and V. G. Yakhno, Autowave Processes in Kinetic Systems: Spatial and Temporal Self-Organization in Physics, Chemistry, Biology, and Medicine, Math. Appl. (Soviet Ser.) 11, D. Reidel, Dordrecht, The Netherlands, 1987.

[41] T. M. Wasylenko, J. E. Cisternas, C. R. Laing, and I. G. Kevrekidis, Bifurcations of lurching waves in a thalamic neuronal network, Biol. Cybern., 103 (2010), pp. 447-462.

[42] A. T. Winfree, When Time Breaks Down: The Three-Dimensional Dynamics of Electrochemical Waves and Cardiac Arrhythmias, Princeton Univ. Press, New Jersey (1987).

[43] A. T. Winfree, The Geometry of Biological Time, Springer-Verlag New York, Second edition (2001).

[44] S. Wolfram, Cellular automata as models of complexity, Nature, 311 (1984), pp. 419-424. 